\title{
The Game Theory of Politeness in Language: A Formal Model of Polite Requests
}

\author{
Roland Mühlenbernd ${ }^{1}$, Przemysław Żywiczyński² ${ }^{2}$, Sławomir Wacewicz ${ }^{2,3}$
}

1 Ca' Foscari University of Venice; 2 Nicolaus Copernicus University, Toruń; 3 Eberhard Karls University of Tübingen

Unedited preprint (not a quotable final version)

\begin{abstract}
Linguistic Politeness (LP) is a fascinating domain of language, as it directly interfaces with human social behavior. Here, we show how game theory, as a higher-order theory of behavior, can provide the tools to understand and model LP phenomena. We show this for the specific case of requests, where the magnitude of request and the resultant Rate of Imposition are subsumed under a more powerful explanatory principle: alignment of interests. We put forward the Politeness Equilibrium Principle (PEP), whereby the more disalignment there is between the interests of Speaker and Hearer, the more LP Speaker needs to offset the imbalance. In the second part of our paper, we flesh out our ideas by means of a formal model inspired by evolutionary signaling theory, and provide a mathematical proof showing that the model follows the PEP. We see this work as an important first step in the direction of reconciling theories of language with signaling theory, by incorporating language into more general models of communication.
\end{abstract}

Key words: linguistic politeness, game theory, polite requests, rate of imposition, alignment of interests, politeness equilibrium principle, evolutionay signaling theory

Funding: SW was supported by a Bekker fellowship from the Polish National Agency for Academic Exchange.

\section{Introduction}

Our study addresses one of the fundamental questions in linguistic pragmatics: Why do speakers use dramatically different utterance formats to express essentially the same propositional content and the same type of illocutionary force (Searle, 1969; Searle \& Vanderveken, 1985)? Take a look at the classic examples:

(1) Pass the salt!

(2) Could you pass the salt, please? (Searle, 1975$)^{1}$

both of which express the same proposition 'Hearer passing the salt in the near future' and the same type of illocutionary force - the directive whereby the speaker wants the hearer to pass the salt in the near future. (1) has the format of an imperative; (2), with the interrogative and the particle 'please', has the format characteristic of conventionally indirect speech acts (in this case, of a polite request; Searle, 1975).

The question spelt out above addresses problems discussed in Gricean and post-Gricean pragmatics, related to the operation of conversational implicature (e.g. (2) flouts the maxim of relevance; Grice, 1975) or the cognitive and communicative principles of relevance (the interpretation of (2) as a polite request produces the greatest positive cognitive effect with the least cognitive effort;

\footnotetext{
${ }^{1}$ Adapted from "Can you pass the salt" - one of Searle's classic examples in the paper "Indirect speech acts" (1975).
} 


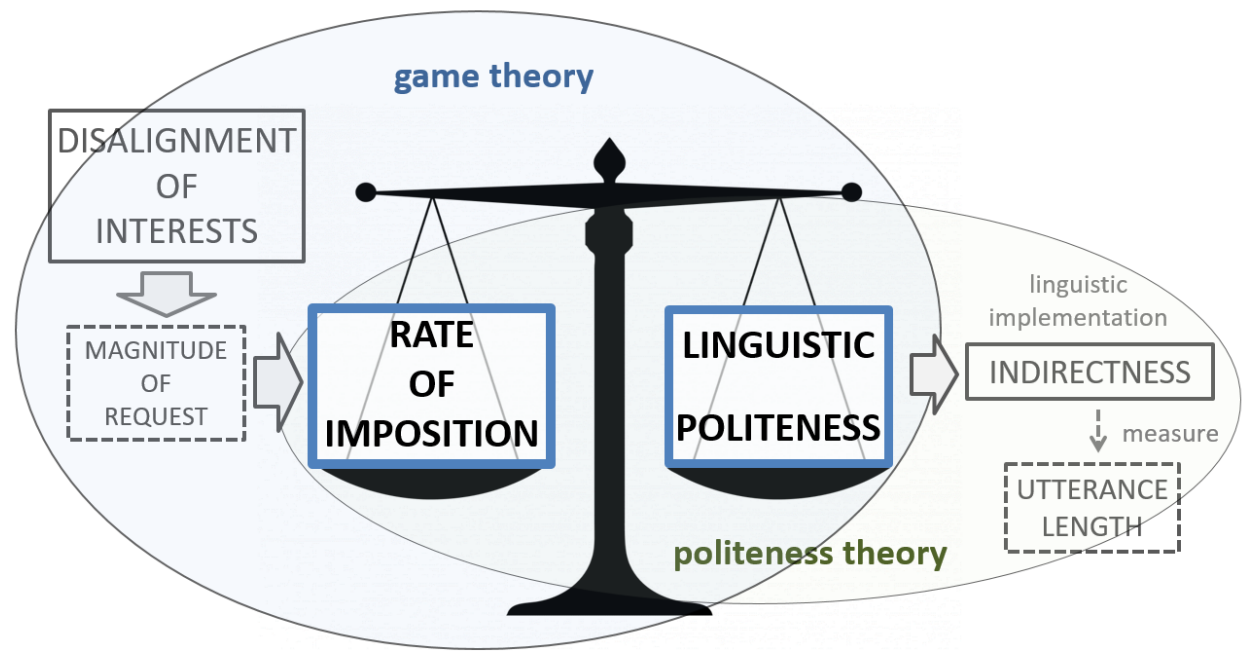

Figure 1: A schematic presentation of the basic assumptions of our work. Game theory, as a higher order theory of behavior, provides tools for understanding and modeling Linguistic Politeness (LP) phenomena. This is illustrated here for the speech act REQUEST. The Politeness Equilibrium Principle (PEP) we propose in Section 3 states that the disalignment of interests between interactants must be offset by the equivalent amount of LP: the greater the disalignment of interests, the more LP is needed. In the case of requests, the degree disalignment of interests is reflected in the magnitude of a request, which in turn translates into the Rate of Imposition (Brown \& Levinson, 1987). Consequently, in making a request, Speaker must offset the Rate of Imposition with a proportional amount of LP. LP is implemented linguistically by means of indirectness (among other ways). Ceteris paribus, indirectness correlates with the length of utterance, which provides a first-approximation measure of LP with a view to making the predictions of the game theoretic models testable.

Sperber \& Wilson, 1986). As already noted, it is also part and parcel of Speech Act Theory, particularly of various accounts of speech act indirectness (e.g. Searle, 1975; Gordon \& Lakoff, 1975; Ervin-Tripp, 1976; Brown \& Levinson, 1978, 1987; Leech, 1983)). In line with the last three sources, the question posed in this article specifically belongs to the area of linguistic politeness (LP).

LP theorists (e.g. Lakoff, 1975; Leech, 1983; Watts, 2003; Eelen, 2001) insist that LP is an essentially linguistic phenomenon; however, its theoretical appeal, and of its models, lies in the fact that it interfaces linguistic behavior and more generally human social behavior (Wacewicz et al., 2015). Currently, the most influential approach to understanding the latter is behavioral game theory, which delivers (i) formal frameworks that allow to model interactive behavior between individuals and (ii) solution concepts - mostly based on rationality assumptions - for explaining particular behavior in specific situations. Our research starts from observing the striking similarity between the core explanatory principles in LP and behavioral game theory (cf. Figure 1); specifically, we believe that (behavioral) game theory as a higher-order theory of behavior can express the basic notions of certain LP models, making them more generalizable and testable. This undertaking is part of a larger project of using game theory to model language behavior. In this we follow existing efforts such as game-theoretic explanations of speech-act indirectness (Pinker et al., 2008) or pragmatic phenomena, particularly in Gricean pragmatics (Franke, 2009).

Our current investigation is limited to the Speech Act Request, exemplified by the utterances (1) and (2), but we think that a model to be proposed here is extensible to other speech acts (see discussion in Section 11). Working from the core assumption of both game theory and influential models of LP, we propose that: 
(A) formats of requests depend on the degree of alignment of interests between Speaker and Hearer ('Politeness Equilibrium Principle', see Section 3).

We develop (A) by suggesting (a) an 'ultimate' mechanism; (b) a proximate mechanism; and (c) a measure, all controversial but well grounded in existing literature:

(a) LP is a social currency that can offset the disalignment ${ }^{2}$ of interests between Speaker and Hearer, when the disalignment is stated in behavioral-economics terms (Werkhofer, 2005; Kaplan, 1999; Danescu-Niculescu-Mizil et al., 2013, - see Section 4); in the specific case of requests, the disalignment of interests is reflected by the Rate of Imposition (how much Speaker imposes on the Hearer - Section 5)

(b) the amount of LP of an utterance is reflected by other variables, of which we focus on indirectness (Brown \& Levinson, 1987, - see Section 6)

(c) indirectness is approximated by utterance length (see Section 5), which leads to the postulate about a prominent relation between LP and utterance length (Zahavi and Zahavi 1997; (Östman, 1989, - see Section 6) ${ }^{3}$

In the second part of our paper, we flesh out our ideas by means of a formal model inspired by evolutionary signaling theory (Section 7), particularly by a principle found in animal communication that we dubbed the 'neediness principle' (Section 8). By focusing on a specific type of speech act (request), our formal model - the 'Politeness Equilibrium Principle' (PEP) model - predicts that Speakers strategically use the optimal amount of LP in requesting favors from Hearers (Section 9). We deliver a mathematical analysis to prove that the PEP model makes correct predictions (Section 10), and discuss some aspects of the model that call for extensions (Section 11).

\section{Theorizing Politeness}

Politeness research now forms a rapidly developing field of study that addresses the problem of politeness holistically (e.g. including both polite and impolite acts; Watts, 2003; Bousfield, 2008; Culpeper, 2011), giving considerable attention to methodological considerations (such as the distinction between Politeness 1 and Politeness 2$)^{4}$ and culture-specific character of politeness phenomena. ${ }^{5}$ In this work, we understand politeness in much narrower terms: following Searle (1975), we take the use of a particular utterance format to express a Speech Act, such as a request, to be dictated by Speaker's choice of how polite she wants her utterance to be. This problem may not lie at the center of recent politeness research, but constitutes the core element of classic politeness theories proposed by Lakoff, Leech, and Brown and Levinson.

To explicate this problem, Lakoff and Leech both appeal to Grice and propose conversational maxims that operate in conjunction with Grice's Principle of Cooperation and his maxims of Quality, Quantity, Relevance and Manner (Grice, 1975). They both understand the Gricean component as serving to gear language use towards maximal clarity of expression; further they both

\footnotetext{
${ }^{2}$ Note that we use the notions alignment and disalignment as complements that describe the same entity from different ends of a scale. By using both terms as a measure, alignment and disalignment are fully inversely proportional, e.g. the more interests are aligned, the less they are disaligned to the same amount, and vice versa.

${ }^{3}$ We concede that this is a statistical relationship, possibly epiphenomenal rather than causal.

${ }^{4}$ Politeness 1 represents the commonsensical notion of politeness; Politeness 2 constitutes the second order, formal concept of politeness elaborated by theorists of politeness (Eelen, 2001: 30-31). Politeness researchers are now for a large part engrossed in questions about the relation between these two types of politeness, and how they should be incorporated into explanations of politeness (e.g. Vilkki, 2006; Terkourafi, 2011; Jucker \& Taavitsainen, 2013).

${ }^{5}$ This trend was ushered by socio-culturally and ethnographically minded accounts of politeness phenomena in different cultures (such as the Chinese 'limao': Gu, 1990; Mao, 1994; Lee-Wong, 1999) and historical reconstructions of the very notion of politeness (Ehlich, 2005).
} 
see politeness as working against the Gricean logic: politeness serves to strategically decrease the clarity of expression in order to make an utterance socially appropriate - to reduce social friction in Lakoff's terms (1975) or to avoid conflict and promote interpersonal comity in Leech's (1980b). Lakoff builds both the Gricean maxims and politeness into the overarching conception of pragmatic competence (analogous to Chomsky's grammatical competence), which specifies the rules for producing pragmatically well-formed utterances (Lakoff, 1973). On Leech's account, the interpretation of utterances depends on the interplay between Grice's Cooperative Principle (CP) and Politeness Principle (PP): the more an utterance is in line with CP, the less it is in line with PP, and vice versa (Leech, 1983: 83-84). Both principles form what Leech calls 'interpersonal rhetoric', which in his model is an element of pragmatics responsible for transmitting information about the illocutionary force of utterances (Leech, 1983: 61). While CP serves to communicate the intended illocutionary force of an utterance (i.e. whether it is an assertive, directive, etc.), the goal of PP is to communicate this illocutionary force in the way that helps maintain good social relations (Leech, 1983: 40). PP subsumes the operation of the Tact Maxim (Leech, 1977, 1980a) (in Leech, 1983 subdivided into six maxims: Tact, Generosity, Approbation, Modesty, Agreement and Sympathy), which is understood in essentially economic terms: the Tact Maxim is used to maximize benefit to Hearer in the case of inherently polite speech acts (such as promises) or minimize cost to Hearer in the case of inherently impolite speech acts (such as requests; Leech, 1983: 109-110). Leech primarily understands LP as speech act indirectness, and the degree of indirectness (reflected by the number of exponents of indirectness, such as the interrogative, modality, negative, preterit forms, etc.) is taken by him to be positively correlated with the amount of politeness (Leech, 1983: 108).

Finally, Brown and Levinson $(1978,1987)$ couch their theory in constructivist terms. Its foundational element is an idealized Speaker - a rational Model Person (see Section 3) - who is able to use language to forward the accomplishment of her goals and is equipped with the two types of wants: to be appreciated by others and to be unimpeded by them (Brown \& Levinson, 1987: 58). Important here is the concept of Face as social commodity taken from Goffman (e.g. (1967)), where it refers to "the positive social value that a person effectively claims for himself by the line others assume he has taken during a particular contact" (Goffman, 1967: 5) and which can be, on the one hand, gratified and, on the other hand, compromised and lost in effect of interacting with others (Goffman, 1967, 1983). Brown and Levinson reinterpret Goffman's idea in psychological terms and understand Face in terms of the two types of wants (i.e. face-wants), designated as positive and negative Face (Brown \& Levinson, 1987: 62). ${ }^{6}$ Similarly to Lakoff, they view interaction as inherently conflictual and see the function of politeness as minimizing threats that interaction causes to the satisfaction of positive and negative face-wants (which are hence designated as Face Threatening Acts, FTAs). The weight (W) of an FTA is taken to depend on three social variables: $\mathrm{P}$ (the perceived difference in power between Hearer and Speaker), D (the perceived social distance between them), and $\mathrm{R}$ (the rate of imposition that a given FTA entails, i.e. how threatening a particular FTA is in a specific culture; Brown \& Levinson, 1987).

\section{The Politeness Equilibrium Principle}

Following Brown and Levinson (1987), but also in line with Leech (1983), we take Speaker's choices to use more or less polite utterance formats to be rational, i.e. to be dictated by goals Speaker

\footnotetext{
${ }^{6}$ Brown and Levinson's theory of politeness has often been criticized for using culture-specific constructs as allegedly universal principles of human behavior (see e.g. Eelen, 2001: 20-29; Watts 2003: 101-116). In this context, their psychological interpretation of Face turned out the most strongly contested claim, with the critics arguing that Brown and Levinson's stress on individualism may hold for behavioral norms in the Western culture but is not representative of other cultural milieus. In fact, the studies of non-European politeness systems, such as the Chinese limao, show that Face can be construed not individualistically but communally (see Footnote 4). Although we follow some predictions of Brown and Levinson's theory (see Section 5), for the reasons spelt out here, we do not subscribe to their conceptualization of Face as necessarily an individual's property.
} 
seeks to accomplish. Brown and Levinson build rationality into the concept of Model Person; accordingly, Model Person is endowed with not only two types of Face-wants (see above) but also rationality:

All our Model Person (MP) consists in is a willful fluent speaker of a natural language, further endowed with two special properties - rationality and face. By 'rationality' we mean something very specific - the availability to our MP of a precisely definable mode of reasoning from ends to the means that will achieve those ends. (1987: 58)

In their model, rationality describes Model Person's ability to calibrate the use of politeness strategies (i.e. the strategies of going Bald-on record, of using positive or negative politeness) to the weight of an FTA. With regard to our research question, we have the following hypothesis: Speaker's behavior is rational in the sense that the amount of linguistic politeness (essentially, the choice of a particular utterance format) she uses negatively correlates with the degree that Speaker's and Hearer's goals are aligned - the less Speaker's goal expressed in a speech act is aligned with Hearer's goal, the more linguistic politeness is expected in Speaker's utterance. Therefore, Speaker's rationality consists in the ability to use an optimal amount of LP for the degree of alignment between her own and Hearer's goals - neither too little, nor too much.

We term such a pattern of language use as Politeness Equilibrium Principle (PEP), and we assume every rational language user to behave in accordance with it. In brief terms, our PEP hypothesis is as follows:

Hypothesis 1 (PEP Hypothesis) The amount of linguistic politeness Speaker uses in a request corresponds negatively to the alignment of interests between Speaker and Hearer: the less aligned the interests are in favor of Speaker, the more linguistic politeness she uses.

\section{Linguistic Politeness as a Social Currency}

Leech's as well as Brown and Levinson's models of LP on which the PEP hypothesis is founded assume that the relation between alignment of interests and politeness is specifically an economic one. That is, not only does the (dis)alignment of interests co-vary with politeness, but it does so economically: Speakers use the currency of LP to pay Hearers proportionally to the degree of disalignment. Requests imply cost to Hearer and benefit to Speaker, so their interests are disaligned as a default, necessitating compensation in LP. In Leech (1983), when making a request, Speaker is obligated to use Tact, Generosity, Approbation, Modesty, Agreement and Sympathy, which act to minimize cost to Hearer. Brown and Levinson define a 'Weight' of a Face-Threatening-Act, which needs to be offset (i.e. paid for) with a commensurate amount of LP; in particular, the greater R - rate of imposition - the more LP is expected in exchange.

The underlying metaphor here is LINGUISTIC POLITENESS IS MONEY. This means that when a given language and culture marks a particular expression as polite, this expression can effectively function in that language/culture in a quasi-economic way, i.e. can be transferred between agents in exchange for goods and services. In other words, LP is conceptualized as a kind of social commodity, or currency, with real, extralinguistic purchasing power. This conceptualization is often explicit, for example Kaplan (1999) observes that "people desire to be paid respect" and identifies honorifics and other politeness markers, such as 'please' as "the coin of that payment" (see Danescu-Niculescu-Mizil et al. 2013). Along similar lines, Werkhofer (2005) - drawing on Simmel's (1900) Philosophie des Geldes - states that despite being a social construct, politeness, like money, can itself motivate and structure courses of action. Like the value of currencies, the value of polite expressions can fluctuate according to the change in the social order, and like monetary resources, one can use linguistic resources to pay what is due in a given socio-communicative context. 
Building on Werkhofer, Watts (2003: 142-144, 151-152) proposes the idea of linguistic capital. Money is a surrogate good that can be exchanged for material, cultural or social goods, and so linguistic capital is another type of surrogate good that can be likewise exchanged. On this conceptualization, language refers to language resources, which "include control over language varieties (e.g. dialect, sociolect, regional standard, national standard, etc.) and/or skilled use in a variety of communicative media (e.g. various form of written media..., or forms of oral media...)" (Watts, 2003: 151-152). They allow their possessor to bargain for material, cultural and social goods. A key term in Watt's theory, politic behavior, is such use of linguistic capital that is construed by participants as paying appropriately, neither too little nor too much.

Like all useful analogies, the above ones also have their limits. When compared specifically to money, LP is non-transferable and not a very precise unit of accounting. And when compared to a valuable resource in general, the stores of LP are not depleted by using it. This key difference is particularly visible from the game-theoretic perspective we espouse here, and makes the extralinguistic value of LP a major puzzle. As Wacewicz et al. noted: "The stability of politeness as an apparently cheap but beneficial strategy is hard to explain unless we assume some kind of inbuilt safeguard against its manipulative use - some cost that is incurred to the polite individual" (Wacewicz et al., 2015: 91). Consider bargains, which imply a balanced transfer of extralinguistic (economically quantifiable) resources in both directions - for example "I'll pay you $\$ 5$ if you give me your pen". Here, complying with the proposed course of action is well explained by the calculation of gains and losses of the conversants, and the disalignment of interests is compensated both ways with real-life resources. This is not the case in requests, such as "Please give me your pen", if Hearer gets no goods or services in return. LP models predict "paying" for the imbalance with an adequate amount of the currency of LP, but behavioral economics predicts immediate "inflation" of that currency. Since the temporal and energetic costs of producing linguistically polite requests are negligible, and the benefits of having the request granted are not, this predicts that:

- Speakers will always use maximum politeness to request maximal possible favors;

- Hearers will never grant a request, and will generally disregard all LP.

However, this does not happen; language users do not always use maximum politeness (in requests and otherwise), but continue to distinguish between more or less polite requests and continue to attach non-trivial value to the level of politeness in a request (and polite language in general - e.g. expressions of gratitude, Fox et al., 2015). This strongly suggests that polite requests are stabilized by some other type of cost beyond the bare production costs. In other words, the mere expenditure of time, energy and cognitive resources on the part of Speaker is not sufficient to increase the level of politeness $\backslash$ indirectness in and of itself, but rather that by doing this, Speaker also pays some other cost.

So, what gives LP its purchasing power? Social status, or social prestige, is one candidate solution - LP can serve as a marker of the status difference between Speaker and Hearer, because all other things being equal, "politeness is inversely correlated with social status" (Danescu-NiculescuMizil et al., 2013). In our specific case, making a request decreases the status of Speaker (requester) and increases the status of Hearer (requestee) proportionally to the LP used in making a request. Zahavi and Zahavi (1997: 75) have proposed this to be a general property of human and non-human communication: "the act of petitioning is costly to the requester. It also decreases the standing or prestige of the requester, and increases the standing of the giver, in the eyes of witnesseswho are more likely to notice a longer request, and to note its significance." (cf. also Quinley 2012: 230). A different possibility, also latent in Zahavi and Zahavi's (1997) account ${ }^{7}$, is that

\footnotetext{
7 "The point of making a request is to get something that is of value to or demands an effort from the giver. After all, if the thing were valueless to its owner, it could be taken without any fuss. Logically, the giver will consent to give up the valued object or spend the effort only if it either cares about the petitioner or gains from the act of
} 
requests are implicit commissives (i.e. it is implied that if a request is granted, Speaker owes Hearer something in return), with LP serving to evaluate the size of the favor. Here, the amount of LP used in asking a favor would put a "price tag" on the favor granted, committing Speaker to later reciprocate proportionally to how much LP was invested into the request. These two accounts, while non-exclusionary, generate different empirical predictions:

(a) perceived value of an LP act will increase proportionally to the number and status of its witnesses;

(b) Speaker feels indebted not in proportion to the size of the favor granted but rather to the amount of LP used in request.

Here, we want to emphasize that we do not commit ourselves to a particular account of the underlying cost of using LP, which must be determined via empirical examination. As Wacewicz et al. established, "[t]o date... no account of the costs of polite linguistic behavior has been offered that could be considered definitive" (Wacewicz et al., 2015: 92). For our present purposes it is sufficient to merely register the fact that LP functions in quasi-economic ways, with an equilibrium between the values spent and gained - a fact implicitly recognized by speakers, and implicitly or explicitly reflected in major theoretic models of LP.

\section{Request Length and Rate of Imposition}

We envisage this model as in principle applicable to most if not all speech acts. In this article, however, we focus only on one speech act type - REQUEST. Generally speaking, directives are easy to understand in terms of alignment of Speaker's and Hearer's interests, while requests i.e. directives that involve transfer of goods and services from Hearer to Speaker (Żywiczyński, 2010) - are particularly amenable to such a conceptualization, and for this reason they seem a good starting point for the construction of our model.

We adopt Searle's (1975: 71) definitions of requests as speech acts meeting the following felicity conditions:

(a) Preparatory condition: Hearer is able to perform action A.

(b) Sincerity condition: Speaker wants Hearer to do A.

(c) Propositional condition: Speaker predicates a future act A of Hearer.

(d) Essential condition: the utterance counts as an attempt by Speaker to get Hearer to do A.

Since the focus of our research is on strategic use of different request formats, we opt for a broader definition of the speech act Request than is usually given in literature. Accordingly, REQUEST is not limited to an individual utterance that Speaker uses to express it (e.g. Searle, 1975; Saeed, 1997) but extends to any previous utterance that is used by Speaker to introduce an utterance in which REQUEST is expressly present as well as to any following utterance in which REQUEST is repeated. In Conversation Analysis, the former are referred to as pre-sequences, in our case pre-requests (Atkinson \& Drew, 1979; Levinson, 1983), and they often serve to check if the conditions for performing a request obtain. For example, in (3):

(3) A: Do you have any money?

giving. The potential giver already knows how it feels about the requester and about the item asked for; what the giver does not know and needs to find out is the importance of the requested object or action to the requester. The more highly the petitioner values the item or action, the more the giver gains by handing it over." (Zahavi and Zahavi (1997): 75). 


\begin{tabular}{ll}
\hline Exemplary Request Length & Examples \\
\hline Length 0 & Say nothing (signal no intention) \\
Length 2 & "Do $X$ !" \\
Length 9 & "Could you be so kind and do $X$, please?" \\
Length 18 & "Listen, I need your help. It is really an emergency case. Could \\
& you please, please, please do $X ? "$ \\
Length $>18$ & "How long do we know each other? We have been having a \\
& great time together. You are a great person. I would like to \\
& collaborate/spend the rest of my life with you. Will you $X$ ?" \\
\hline
\end{tabular}

Table 1: Requests ordered by exemplary utterance length.

B: Yes.

A: Could you lend me a few coins?

A's pre-request serves to check if the preparatory conditions obtains, i.e. whether B is able to perform action A. But on our account, we also classify as parts of requests utterances that do not pertain to the felicity conditions of REQUEST but are strategically used by Speaker to express a request or increase the chances of it being granted by Hearer; these include begging, paying Hearer compliments, showing interest in Hearer or courting Hearer (cf. Brown \& Levinson, 1987, for the taxonomy of positive politeness strategies). Many of them represent strategies whereby Speaker to present her request - uses a number of utterances or a number of conversational turns. ${ }^{8}$

Based on the assumption about length and politeness, we predict one-utterance requests to be less polite than those that take up a number of utterances, e.g. by involving pre-requests as in (3) (cf. Table 1 for the exemplary Request Length 18). We follow Östman's (1989) work on the relation between utterance length and politeness and link the amount of politeness of a request to the number of lexical items that this request is comprised of. Thus, among one-utterance requests, their length and amount of politeness will differ depending whether they employ the minimal structure characteristic of direct commands, such as (1) (cf. Table 1 for the exemplary request length 2: "Do X!" - consisting of 2 lexical items) or contain exponents of speech act indirectness, such as (2) (cf. Table 1 for the exemplary Request Length 9: "Could you be so kind and do X, please?" - consisting of 9 lexical items). Importantly, to evaluate the amount of politeness in a request we need to look at the overall utterance length (operationalized as a number of lexical items), which may comprise an individual utterance, as in exemplary Request Length 2 and 9, but also a number of individual utterances, as in the exemplary Length 18 (cf. Table 1).

Next, requests should also be assessed depending on request magnitude, or to use Brown and Levinson's term, Rate of Imposition. A crucial point to observe is that Rate of Imposition is easily translated into alignment of interests: Rate of Imposition is a term in politeness theory that, in the specific context of requests, expresses the disalignment of interests between Speaker and Hearer in disfavor of Hearer. Again for exemplary purposes we categorize different Rates of Imposition from small to great in the following way: (i) joint task, (ii) small favor, (iii) big favor, (iv) tall obligation, and (v) presumptuous demand. The above degrees of Rate of Imposition represent different degrees of how disaligned (in favor of Speaker) Speaker's and Hearer's goals are, from completely aligned to extremely disaligned (cf. Table 2).

By intuition different Rates of Imposition require appropriate Request Lengths (cf. Table 1 for the exemplary Request Length 0). To say nothing of course doesn't help at all. A direct command (cf. Table 1 for the exemplary Request Length 2) is suitable for joint tasks. Indirectly formulated requests (cf. Table 1 for the exemplary Request Length 9) are appropriate for a small favor. While big favors, i.e. such that incur a significant cost to Hearer, often require longer-lasting strategies,

\footnotetext{
${ }^{8}$ In principle, these can even be distributed over a number of distinct interactions.
} 


\begin{tabular}{ll}
\hline Request type & Examples \\
\hline Joint task & Pass me the ball (team mate), let's finish joint project, ... \\
Small favor & Open the window, take out the trash, ... \\
Big favor & Lend me 500 Euro, pay our restaurant bill, ... \\
Tall obligation & Marry me, fund my start up business... \\
Presumptuous demand & Give me all your money, be my slave for two years... \\
\hline
\end{tabular}

Table 2: Different request types ordered by Rate of Imposition (magnitude of the favor).

such as e.g. begging and the like, that can go beyond the length of an individual utterance (cf. Table 1 for the exemplary Request Length 18). To convince the Hearer of a tall obligation usually needs strategies that are even more longer-lasting, involve a long preface or even vocal courting for days and weeks (cf. Table 1 for going beyond the Request Length 18). For presumptuous demands, such as presented in Table 2 (one can generally think of them as being threats) - linguistic means alone are insufficient, since hardly anybody would give Speaker all her money only because Speaker has made a very long request: one would need more than merely words. This is an important point, since it shows that LP can be used to pay compensation in proportion to the Rate of Imposition, but only up to a specific magnitude.

It has not probably gone unnoticed that our understanding of request magnitude as Rate of Imposition disregards other variables crucially important to the evaluation of request magnitude. These include, most importantly, the two other factors $\mathrm{P}$ (the perceived difference in power between Speaker and Hearer, or status) and D (the perceived social distance between Speaker and Hearer, or familiarity) that Brown and Levinson use, together with Rate of Imposition, to determine the Weight of a Face Threatening Act (1987; see Section 2). We have decided to abstract from these variables for modeling purposes: we want to show that when keeping all the other variables constant, the Rate of Imposition of a request determines the amount of LP used by Speaker (operationalized here as Request Length; see Section 12).

Furthermore, on our conceptualization, Rate of Imposition does not have to be a static entity, entirely dependent on what is being requested (e.g. a pen vs. a car) - a point in Brown and Levinson's model that is often criticized, for example by Watts (2003 114; although his criticism mainly concerns the Power and Distance variables, it is easy to extend it onto Rate of Imposition). We want to stress that that the precise calibration of the Rate of Imposition expressed in a request is contingent on a specific interactional context in which Speaker and Hearer are engaged. The important facets of this context are identified by Curl and Drew in their work on requests (2008), when they write about Speakers entitlement to make a specific request to a specific Hearer as well as Speakers understanding of the contingencies related to granting a request (e.g. Speakers evaluation of the likelihood of Hearers granting a request). In consonance with Curl and Drew (2008), we take these factors to influence the Rate of Imposition of a request and bear on the amount of LP used when formulating it.

To revert to more general concerns, note that the mapping between Rate of Imposition and amount of LP understood as Request Length displays a clear relationship: the greater the Rate of Imposition of a request type, the greater the Request Length. By taking Request Length as an indicator for Speaker's amount of LP she uses (cf. Section 6), and by assuming that the Rate of Imposition of a request type represents the divergence between Speaker's and Hearer's utility - or in other words their degree of disalignment - then the following relationship emerges: the less aligned interlocutor's goals are in a request type, the more LP is present in a request form used by Speaker. Furthermore, by taking LP as a linguistic commodity or currency, the use of LP constitutes a cost for the Speaker: the more LP she uses, the more costs she incurs.

Furthermore, this mapping is in line with the PEP hypothesis, extended by the additional assumption that if the Rate of Imposition of a request is too big, then the use of LP alone does not 
suffice to forward such a request. In such cases, some extralinguistic material is necessary ${ }^{9}$ and as we will delineate later - the best strategy is to forgo the use of LP at all. The nature of the mapping between Request Length to different request types, with the additional assumption that Request Lengths indicate the amount of LP the speaker uses, can be formalized as the Politeness Equilibrium Principle in the following way:

Definition 1 (The Politeness Equilibrium Principle (PEP)) Given a situation where Speaker requests goods or services from Hearer quantified as a value of Rate of Imposition $r \in \mathbb{R}^{+}$. Let's define function $L P: \mathbb{R}^{+} \rightarrow \mathbb{R}^{+}$as the amount of linguistic politeness used in requesting $r$. Then Speaker follows the PEP by using an optimal strategy LP* $: \mathbb{R}^{+} \rightarrow \mathbb{R}^{+}$, iff for any two Rates of Imposition $r, r^{\prime} \in \mathbb{R}^{+}$the following two conditions hold:

(1) $\forall r \geq r_{\delta}: L P^{*}(r)=0$

(2) $\forall r, r^{\prime}<r_{\delta}$ : if $r>r^{\prime}$ then $L P^{*}(r)>L P^{*}\left(r^{\prime}\right)$

whereby $r_{\delta} \in \mathbb{R}^{+}$is the lower threshold of a Rate of Imposition of a request that cannot be paid for by linguistic politeness (or linguistic means in general) alone.

Informally, the two conditions of Definition 1 state that i) for situations where Speaker's request is of an unreasonable amount $\left(r \geq r_{\delta}\right)$ the best choice is to say nothing at all, and ii) for situations where Speaker's request is of a reasonable amount $\left(r<r_{\delta}\right)$ the optimal amount of LP is a strictly monotonously increasing function of Rate if Imposition $r$. In Section 7 we will develop a gametheoretic model based on ideas from signaling theory, which delivers a possible explanation for the stability of the PEP in human language. But before, we will discuss in more detail the relationship between utterance length and the amount of linguistic politeness - particularly with reference to indirectness of speech.

\section{Indirectness and Utterance Length}

The way the PEP is formulated requires quantifying linguistic politeness. As already noted, we assume that utterance length is positively correlated with the amount of politeness it expresses (operationalized as a number of lexical items; Östman, 1989). This correlation is of statistical nature and therefore not infallible, as can be illustrated by the comparison of the following examples:

(4) Pass the salt, honey.

(5) Pass the fucking salt, you fucking idiot.

Our reasoning to link utterance length with the amount of linguistic politeness is the following: speech indirectness (or conventional indirectness; Blum-Kulka, 1987) is one of the commonest ways of expressing linguistic politeness; since indirect utterances tend to be longer than direct utterances, longer utterances tend to be politer than shorter ones. Regarding the first assumption, we are in line with Leech (1983) and Brown and Levinson (1987), who take speech act indirectness and linguistic politeness as equivalent (see Blum-Kulka, 1987, for a critical discussion of this point). This point finds support in empirical studies, mainly Blum-Kulka's research on indirectness and politeness judgments (1987) as well as corpus-based research (e.g. Danescu-Niculescu-Mizil et al., 2013).

The second assumption - about the relation between speech act indirectness and utterance length - is based on the view (expressed e.g. by Searle, 1975 or Leech, 1980a) that the more

\footnotetext{
${ }^{9}$ Extralinguistic matter indicates goods or services that can be exchanged for granting a request, as in "Give me all your money. And I will give you my yacht."; it may also involve a threat, e.g. holding Hearer at gunpoint.
} 
exponents of indirectness in an utterance, the more indirect, i.e. polite, this utterance is. Again, counterexamples are easy to find where length does not necessarily translate into the amount of indirectness, and hence politeness. Consider examples that Leech provides to show how increasing indirectness increases politeness:

(6) Answer the phone.

(7) I want you to answer the phone.

(8) Will you answer the phone?

(9) Can you answer the phone?

(10) Would you mind answering the phone?

(11) Could you possibly answer the phone (Leech, 1983: 108).

On Leech's reasoning, (8) is politer than (7) because using the interrogative instead of the affirmative increases optionality and in this way makes the utterance more indirect; this is so, despite the fact that utterances (8) (consisting of 5 lexical items) is shorter than (7) (consisting of 7 lexical items).

But here too, there is empirical evidence that utterance length, although not infallible, is a stable predictor of how indirect, and hence polite, an utterance is judged to be. This evidence comes mainly from corpus studies (e.g. McEnery et al., 2001; Danescu-Niculescu-Mizil et al., 2013; Diani, 2014) and studies of communication in online communities (e.g. Brennan \& Ohaeri, 1999; Duthler, 2013; Burke \& Kraut, 2014). The statistically robust correlation between length and indirectness in our view explains the results obtained by Östman (1989), who set out to verify Haiman's famous proposal that "the social distance between interlocutors corresponds to the length of the message, referential content being equal" (Haiman, 1983: 783). An artificial language experiment led Östman to the more general conclusion that longer messages are consistently interpreted as more formal, and hence more polite, than their shorter equivalents with the same semantic content. ${ }^{10}$

Last but not least, the decision to use utterance length as an index of the amount of politeness is motivated by the modeling and experimental logistics. Particularly in behavioral experiments, the operationalization of politeness as scale that is easy to measure should prove to be of great benefit. In this respect, it is a better, though perhaps cruder, measure than e.g. the cognitive interpretation of indirectness-qua-politeness as "the relative length of the inferential path needed to arrive at an utterance's illocutionary force" (Blum-Kulka, 1987: 133; cf. Żywiczyński, 2010). We need to stress here that such an operationalization is solely based on probabilistic considerations (i.e. that on average politer utterances tend to be longer than their less polite equivalents) and not on the conviction that there is some direct causal link between length and politeness. For example, we do not believe that politeness is explained by a higher cost of producing longer utterances: it may be true that producing (2) is energetically more costly than (1), but this difference is negligible and cannot explain the difference in politeness effect between the two utterances (see Section 4).

Before engaging in modelling LP, we must address one more important concern, which can be expressed by the question: Are there linguistic forms that are inherently polite? The view that gives the affirmative answer to this question - found e.g. in Brown and Levinson 1987), some of whose ideas we adopt - has been a target of severe criticism by more recent politeness research (see for example Watts's lengthy discussion of formulaic and semi-formulaic expressions of LP; 2003: 168-200). In our case, not just the sources we cite but, more importantly, the operationalization of LP as the degree of indirectness and utterance length strongly suggests that we, just like Brown and Levinson, give the affirmative answer to the question spelt out above. However, in the point

\footnotetext{
${ }^{10}$ See also Zahavi and Zahavi (1997), who point to the relationship between utterance length and Politeness in contraposing human language use to animal communications (cf. Section 8).
} 
of fact, we do not subscribe to such a view, and to explain the exact nature of our position on LP, we appeal to what can be called a Bayesian view of LP. Accordingly, prior uses of utterance formats form beliefs about what constitutes LP, e.g. that polite utterances tend to be more indirect and longer than less polite ones. These beliefs are reflected in interactants' ongoing evaluations of the politeness of utterances; importantly, these evaluations are mitigated by the specificity of interactional contexts in which utterances appear. Current uses may lead to changing future beliefs about LP; we could even imagine that speech act directness and utterance shortness become positively correlated with the degree of LP.

Even then, the Politeness Equilibrium Principle will hold and the model that we present in the forthcoming sections will work, and it will continue to work as long as the two requirement are met:

- politeness is measurable, i.e. the form of an utterance is able to lead us to reliable beliefs about the degree of politeness it expresses; and

- measures of politeness enjoy at least some degree of intersubjectivity, i.e. interactants are able to form similar evaluations of how much politeness is expressed in an utterance;

However, at least in English, the link between indirectness (and hence utterance length) and politeness is extremely robust, to the point of being codified in prescriptive grammars of the English language (see e.g. Swan, 2005: 401, 409-412).

\section{Insights from Signaling Theory and Game Theory}

It is important to note that language use according to the PEP forms a communicative pattern dubbed by Lewis (1969) signaling equilibrium or signaling system ${ }^{11}$ : the sender (Speaker) sends a different signal in each state. ${ }^{12}$ In our model, different states correspond to different Rates of Imposition, and different signals correspond to different amounts of LP. The recognition that a communicative pattern according to the PEP forms a signaling system allows us to attribute the following properties to it: Since a signaling system generally establishes a one-to-one mapping between states and signals, the PEP establishes a one-to-one mapping between Rates of Imposition and amounts of LP. Furthermore, a signaling systems forms a Nash equilibrium (cf. Myerson, 1991) over expected utilities of Speaker and Hearer. ${ }^{13}$ This gives communicative patterns according to the PEP an aspect of stability in usage. Such stability is further formally expressed by the fact that signaling systems represent evolutionary stable strategies (cf. Maynard Smith, 1982): they are resistant against small perturbations of (language) change.

Note that Lewis' definition of signaling systems is based on his model of signaling games representing situations where the interests of Speaker and Hearer are completely aligned. But the request types we are concerned with do generally represent situations where the interests of Speaker and Hearer are not aligned (except joint tasks, cf. Table 2), but where Speaker benefits from a request being granted by Hearer. As already mentioned, the magnitude of such a request is indicated by its Rate of Imposition, which in turn defines nothing less than the degree of disalignment between the objectives of Speaker and Hearer. Theoretical work on game-theoretic signaling models shows

\footnotetext{
${ }^{11}$ Note that this counts solely for all Rates of Imposition $r<r_{\delta}$.

12 Admittedly, this is only one part of a signaling system. The other part entails that the receiver (Hearer) uses an interpretation strategy that is mirroring the sender's (Speaker's) strategy. But we abstract from this part (and implicitly assume that the receiver behaves accordingly), since we exclusively focus on the sender's (Speaker's) behavior in this study.

${ }^{13}$ In informal terms, a Nash equilibrium is a strategy profile which neither interactant has any incentive to deviate from, under the assumption that they are rational in trying to maximize their expected utilities.
} 
that i) signaling system are not evolutionary stable - but tend to collapse by inflation ${ }^{14}$, or by establishing a non-signaling equilibrium (cf. Maynard Smith \& Harper, 2003) - if the magnitude of disalignment between Speaker and Hearer is too large (cf. Crawford \& Sobel, 1982), and ii) small magnitudes of disalignments can still entail evolutionary stable signaling systems if the signals involve different amounts of costs (van Rooy, 2003; Jäger, 2008). ${ }^{15}$ Note that the latter point is in line with the basic assumption that LP is a currency and therefore its use must involve strategic costs; and the former point is in line with the PEP, namely that there is a lower threshold $r_{\delta} \in \mathbb{R}^{+}$ of the magnitude of disalignment for which LP cannot pay off the favor and the best strategy is to say nothing at all for Speaker, and to not respond for Hearer - or in other words, a non-signaling equilibrium (cf. Maynard Smith \& Harper, 2003).

Quinley (2011, 2012) applied a different game-theoretic model for studying polite requests, which is based on the trust game (cf. Lev-On et al., 2010). ${ }^{16}$ This game model includes by definition the juxtaposition of the option i) to make a verbal request and the option ii) not to make it, i.e. to say nothing at all. The rationale behind choosing i) or ii) depends on the degree of trust in Hearer: What is the probability that Hearer will grant my request? If it is very low, then my social/emotional costs (of losing face) in making a request exceed my expected utility of being helped (cf. Quinley, 2012, p. 229). As we will show in Section 9, the same mechanism in our model determines if Speaker makes a verbal request or says nothing at all. The difference between Quinley's model and ours is that our model is based on the signaling game and a principle derived from signaling theory, that allows Speaker not only to decide between making or not making a request or not but also on how much LP should be optimally used in a request. ${ }^{17}$

\section{The Handicap Principle and the Neediness Principle}

To our knowledge, van Rooy (2003) was the first to analyze LP by means of a game-theoretic signaling model. He was able to show that different signaling costs alone are not necessarily sufficient to establish an evolutionary stable signaling system for non-aligned situations. In his model it is also important HOW these costs differ among different speakers. His main hypothesis implies that a polite request is a linguistic manifestation of what in animal communication is known as the handicap principle (Zahavi, 1975). The classic exemplification of the handicap principle is the peacock's tail, the size of which both attracts peahens and makes its owner easy to detect by predators.

The idea is as follows: In a mating situation the interest of the female is to figure out if the male is a high type or a low type, since it has a strong preference to mate with high types. The interest of any male, on the other hand, is to mate with the female, no matter if it is itself a low or high type. Therefore, interests between male and female are disaligned. ${ }^{18}$ Now, the best strategy of a high type male is to send a signal that indicates its high type status. The problem is that a

\footnotetext{
${ }^{14}$ Inflation means that Speaker always signals in the most valuable way regardless of the state she is in, - in terms of Politeness theory - always uses a maximal amount of LP.

${ }^{15}$ Furthermore, Ahern (2015) and Ahern and Clark (2017) show that an intermediate magnitude of disalignment in signaling games can neither lead to a perpetually stable system, nor to a total collapse of the language system, but to a steadily change of it. They use tools from evolutionary game theory to replicate a diachronic linguistic phenomenon of unidirectional and cyclic nature, known as the Jespersen cycle.

${ }^{16}$ See Quinley and Ahern (2012) and Asher and Quinley (2012) for relevant applications of 'extended trust games'.

${ }^{17}$ Note that Quinley's $(2011,2012)$ model involves a similar mechanism in that Speaker has the option to choose how much 'face to pay' to make the request. This is in parallel to the cost function in our model. Nevertheless, Quinley's approach does not explicitly model the usage of different amounts of cost (LP) as different signaling strategies, but shows for what amount of costs (face) it is reasonable i) for Speaker to go forward with a request and ii) for Hearer to grant it.

${ }^{18}$ Note that alignment of interest - the degree to which the interests of interactants overlap or conflict - is a fundamental explanatory principle in (formalizing) animal communication, and can be found to different degrees in different interactive situations. See Searcy and Nowicki (2005) for a thorough compilation.
} 


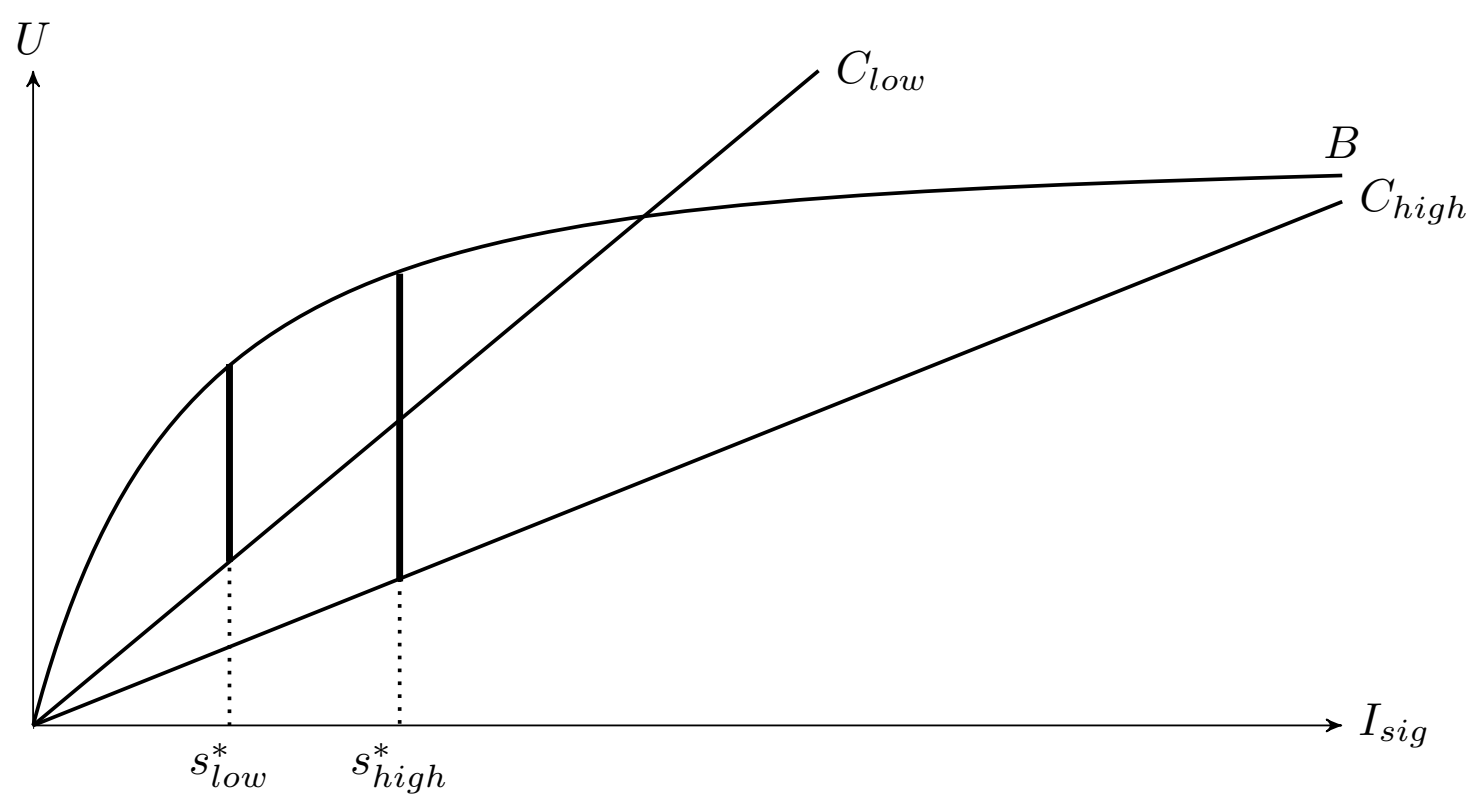

Figure 2: An illustration of the handicap principle, replicated from Johnstone (1997).

low type male can imitate this signal, pretending he is high type, and if the same signal is used by both the low and the high type, it will lose its informative value. A way of making such a signal reliable is to make it is more costly to produce for a low type male than for a high type male.

Johnstone's (1997) study, recapitulated in Figure 2, provides a useful illustration of the handicap principle. It shows that if the cost function for a low type $C_{l o w}$ has a stronger slope than for a high type $C_{\text {high }}$ then the optimal signal ${ }^{19}$ for a low type male $s_{\text {low }}^{*}$ is less intense than the optimal signal for the high type male $s_{\text {high }}^{*}$. As a result, both types produce different optimal signals, a situation that helps the female to distinguish a low type from a high type male.

How can this principle be transferred to human language? The quintessence of this transfer would be that (i) high types produce more polite or complex expressions than low types, and (ii) such expressions are more costly to produce for low than for high types. We think that both assumptions are somehow odd. Let's assume that high type humans differ from low type humans in social status. Under this assumption, it follows that high-status humans are more polite than lowstatus humans, everything else being equal. But is this really the case in human society? Intuitively not. ${ }^{20}$. And the reason is that the second assumption does not seem to apply to humans either: why should it be more expensive for low types to use more polite expressions than for high types? In fact, even van Rooy admits this questionability of his approach at the end of his study. ${ }^{21}$ The value of van Rooy's contribution is the application of signaling theory to the study of the stability of complex communication forms, such as polite requests. But we think that he looked in the wrong place. We argue that another principle that is related to the handicap principle might be a much better starting point for a model that illustrates the stability of the use of LP in requests.

The main problem with the handicap principle is that it assumes that choosing a more or less complex signal depends on the type of Speaker. But looking back to Section 5 , it is more reasonable

\footnotetext{
${ }^{19} \mathrm{~A}$ signal is optimal, if it maximizes the difference of benefit and costs, thus $B-C_{i}$

${ }^{20}$ It is probably exactly the other way around: A relevant observation here is that all other things being equal, "politeness is inversely correlated with social status" (Danescu-Niculescu-Mizil et al., 2013). Thanks also to Robin Clark and Christopher Ahern to point out this divergence.

${ }^{21}$ See also Quinley (2012, p. 226) for further points of criticism.
} 


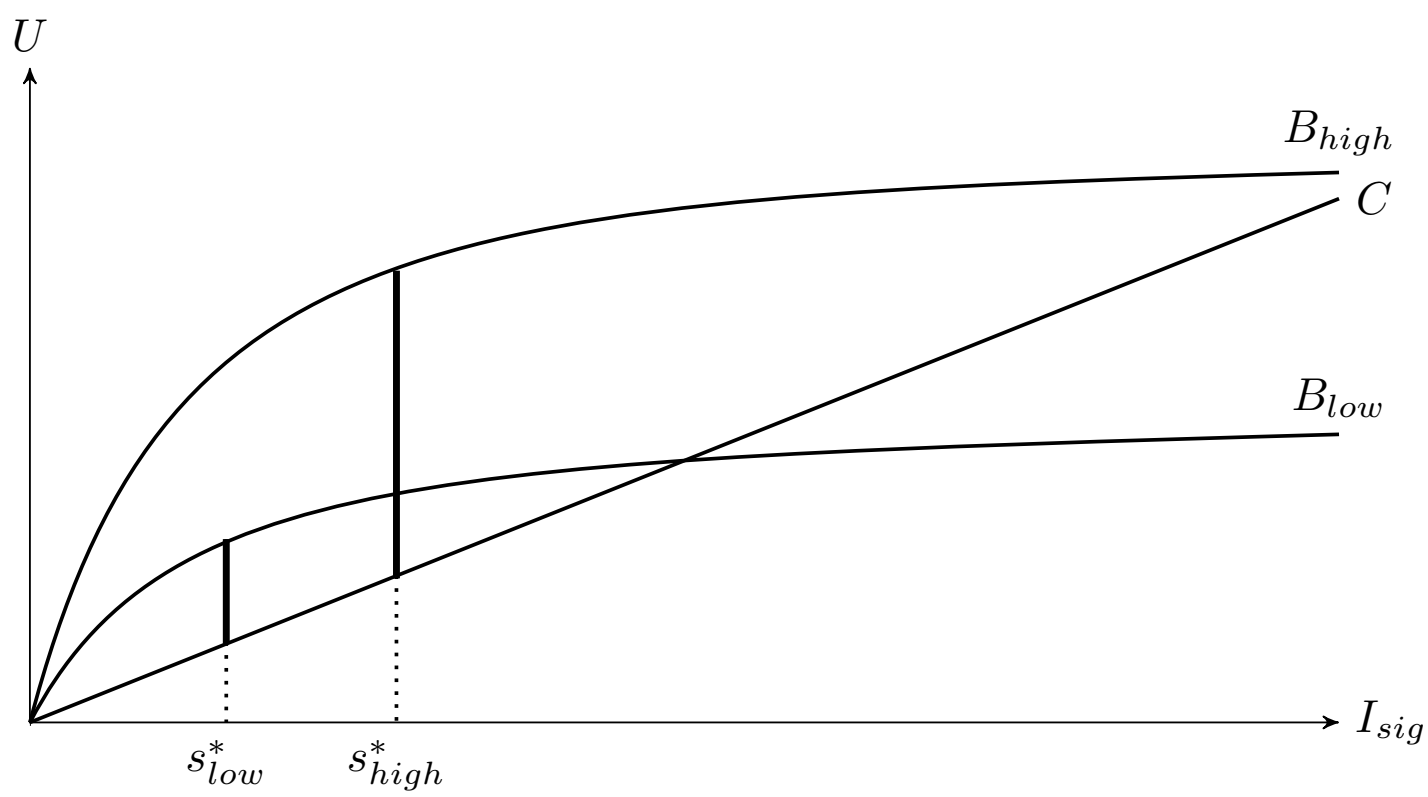

Figure 3: An illustration of the neediness principle, replicated from Johnstone (1997).

to assume that choosing a more or less complex signal depends on the Rate of Imposition of Speaker's request. Such a principle can also be found in animal communication systems. A strikingly similar case is that of offspring that signal their need for food to their parents with begging calls (e.g. Maynard Smith \& Harper, 2003). It is reasonable to assume that the hungrier they are - in other words, the more food they demand - the more intensively they signal their need. In such a case the optimal signal intensity does not inherently differ among the young animals, but rather reflects their need for food. ${ }^{22}$ This scenario was also illustrated by Johnstone (1997) ${ }^{23}$ and recapitulated in Figure 3: here $B_{\text {high }}$ represents the benefit function for a high-need signaler, and $B_{\text {low }}$ for a low-need signaler. The cost function $C$ is invariant among individuals and the optimal signal intensity depends solely on the magnitude of need: the greater the need, the more intense the signal.

Let's call this the neediness principle: the more an individual needs, the more intensive is its signal. It can be shown that this principle enables a stable and reliable communication system, and it seems to be a much better reflection for the PEP. The proposed relationship between the neediness principle in animal communication and the use of Politeness in Human language was already suggested by Zahavi and Zahavi (1997: 75):

The duration of vocalization, like its volume or rhythm, is correlated to its message. The more persuasive a requester feels compelled to be, the greater the duration of his or her request, in any language: "Please"; "Could you please"; "If you really don't mind, could you please" and so on. Likewise, the more nestlings repeat their begging, the more urgent their parents understand the begging to be.

In what follows we use the neediness principle to develop a game-theoretic signaling model in which optimal signaling follows the PEP.

\footnotetext{
${ }^{22}$ Fundamental aspects of the difference between the handicap principle and the neediness principle are also discussed in Maynard Smith and Harper (2003: 17ff.) as 'signals as a handicap' and 'signals as indices'.

${ }^{23}$ Many thanks to Robin Clark and Christopher Ahern, who drew our attention to Johnstone's neediness principle as a good base model for studying linguistic politeness.
} 


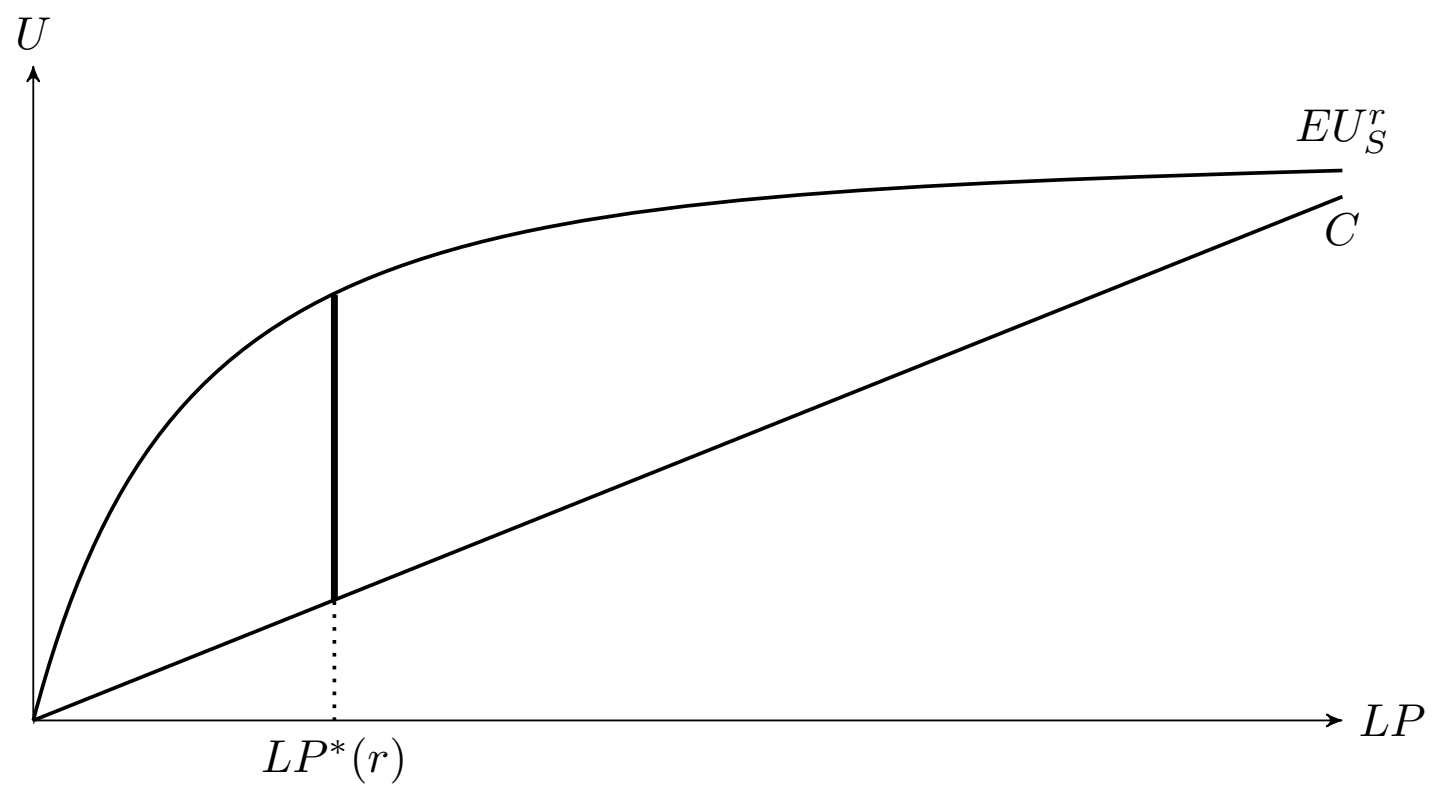

Figure 4: For a request of Rate of Imposition $r$, the $E U_{S}^{r}$-function represents the expected utility for Speaker. The function strictly monotonously increases with the amount of LP involved in the verbal request, whereby the slope decreases. The $C$ function represents Speaker's cost that increases linearly with the amount of LP involved in the verbal request. The optimal choice for Speaker is a LP amount $p$ that maximizes $E U_{S}^{r}(p)-C(p)$. In the given scenario $L P^{*}(r)$ is optimal.

\section{The Politeness Equilibrium Principle (PEP) Model}

The central adoptions and alterations for the transfer of the neediness principle in animal communication to the PEP in human communication are as follows:

(a) While in animal communication systems individuals try to maximize their benefit $B$, Speaker tries to maximize her expected utility $E U_{S}$. Note that $E U_{S}$ involves probabilistic assumptions on Speaker's side, thus takes into consideration that Speaker is able to form probabilistic beliefs about the expected behavior of Hearer.

(b) Signal intensity is measured in terms of the amount of LP in Speaker's utterance (cf. Section $6)$.

(c) Just as the benefit for food begging animals increases strictly monotonously with signal intensity, we assume that the expected utility for Speaker increases strictly monotonously with the amount of LP.

(d) Just as the signal production costs for food begging animals increases linearly with signal intensity, we assume that Speaker's cost for producing an utterance similarly increases linearly with the amount of LP Speaker uses.

Figure 4 explains these relations. The x-axis shows the amount of $L P$ in an utterance. For Speaker's cost function $C: \mathbb{R}^{+} \rightarrow \mathbb{R}^{+}$we assume a linear positive correlation to the amount of LP. Furthermore, let's define (i) $r \in \mathbb{R}$ as the Rate of Imposition of a particular request (or Speaker's reward for that request), and (ii) $\operatorname{Pr}_{H}(r \mid p)$ a probability function for Hearer agreeing to a request of Rate of Imposition $r$, given a specific LP amount of $p$. Then we can define Speaker's 
expected utility for getting a request of Rate of Imposition $r$ granted by producing an utterance with LP amount $p$ as $E U_{S}^{r}(p)=P r_{H}(r \mid p) \times r$. We make an additional assumption that the function $\operatorname{Pr}_{H}(r \mid p)$ strictly monotonously increases with amount of LP $p$, whereby the slope decreases. The idea behind this is as follows: the more LP Speaker uses, the higher the probability that Hearer grants a request of Rate of Imposition $r$. But this effect mitigates over the amount/lengthening of utterances. ${ }^{24}$ Note that the function $E U_{S}^{r}$ has by definition the same shape as the probability function $P r_{H}$, simply because reward value $r$ is a fixed value for the given situation. In other words, the function $E U_{S}^{r}$ strictly monotonously increases with amount of LP $p$, whereby the slope decreases, as displayed in Figure 4. We assume that Speaker and Hearer use LP in a rational way. The Speaker is rational if she uses an amount $p$ of LP that maximizes the difference between expected utility and costs $\left(E U_{S}^{r}(p)-C(p)\right)$. In the scenario of Figure 4 the amount $L P^{*}(r)$ is optimal for a request of magnitude $r$.

Now let's assume that the scenario in Figure 4 represents a situation where the interests of Speaker and Hearer are more or less aligned. Then the model makes the prediction that a comparably low $^{25}$ amount LP is optimal for the request. According to the PEP we would expect the optimal amount of politeness to be greater in case of less aligned interests.

But how would the picture change when interests between Speaker and Hearer are less aligned? If we adopt Johnstone's idea of the neediness principle (Figure 3) then we should expect that the greater the Rate of Imposition $r$ of the speaker, the greater the slope of the $E U_{S}^{r}$-function. But this is not a reasonable assumption for our model. Note that the $E U_{S}^{r}$-function is a product of the value of the request's Rate of Imposition $r$ and probability $\operatorname{Pr}_{H}$ of Hearer to agree to it. On the one hand, we can reasonably assume that if Speaker's request is bigger, accordingly the Rate of Imposition $r$ is greater, which would lift the slope of the $E U_{S}^{r}$-function. But on the other hand, it is also reasonable to assume that the function $\mathrm{Pr}_{H}$ is declined with greater Rate of Imposition $r$, which would accordingly decline the slope of the $E U_{S}^{r}$-function. Taken together, a bigger request might increase the Rate of Imposition $r$ but at the same time decrease the probability $\operatorname{Pr}_{H}$ of the hearer to grant the request.

The following example should make this point clear: let's assume that we have a strictly monotonically increasing function over amount of LP $F: \mathbb{R}^{+} \rightarrow \mathbb{R}^{+}$. Furthermore, let's assume that the probability of Hearer to grant a request of Rate of Imposition $r \in \mathbb{R}^{+}$for Speaker's utterance involving an LP amount of $p$ is defined as $\operatorname{Pr}_{H}(r \mid p)=\frac{F(p)}{r}$. Then it is easy to see that the expected utility function $E U_{S}^{r}(p)=P r_{H}(r, p) \times r=F(p)$ is invariant over $r$. In other words, here the slope of the $E U_{S}^{r}$-function does not depend on the Rate of Imposition $r$, simply because it is to the same degree mitigated by the probability function $\operatorname{Pr}_{H}$. This example shows that given the current model, Speaker's expected utility function $E U_{S}^{r}$ can be invariant over the magnitude of the request; in other words it is possible that $\exists r, r^{\prime} \in \mathbb{R}^{+}, r \neq r^{\prime}: \forall p \in \mathbb{R}^{+}: E U_{S}^{r}(p)=E U_{S}^{r^{\prime}}(p)$. Note that this is an important difference to the food begging scenario, where the magnitude of the request (benefit) determines the slope of the utility function.

This example shows two things. First of all, it reveals that the assumption that a greater Rate of Imposition $r$ implies that the $E U_{S}^{r}$-function has a greater slope is not tenable in the PEP model. And second, it entails that we need another mechanism that alters the $E U_{S}^{r}$-function depending on the value $r$, since otherwise the model does not predict the PEP.

A reasonable solution is not to change the slope of the function, but rather the zero point of the function. This approach assumes that depending on the Rate of Imposition, Hearer expects a minimum level of LP to be offered by Speaker. And such an expectation can be assumed to be a convention or norm in a given society. This can be illustrated by the following dialogue between mother $(\mathrm{M})$ and child $(\mathrm{C})$ :

\footnotetext{
${ }^{24}$ The difference in probability between saying nothing and making the slightest gesture about the intention is much greater than adding another polite request to an hour of courting.

${ }^{25}$ In comparison to less aligned situations, as depicted in Figure 5.
} 


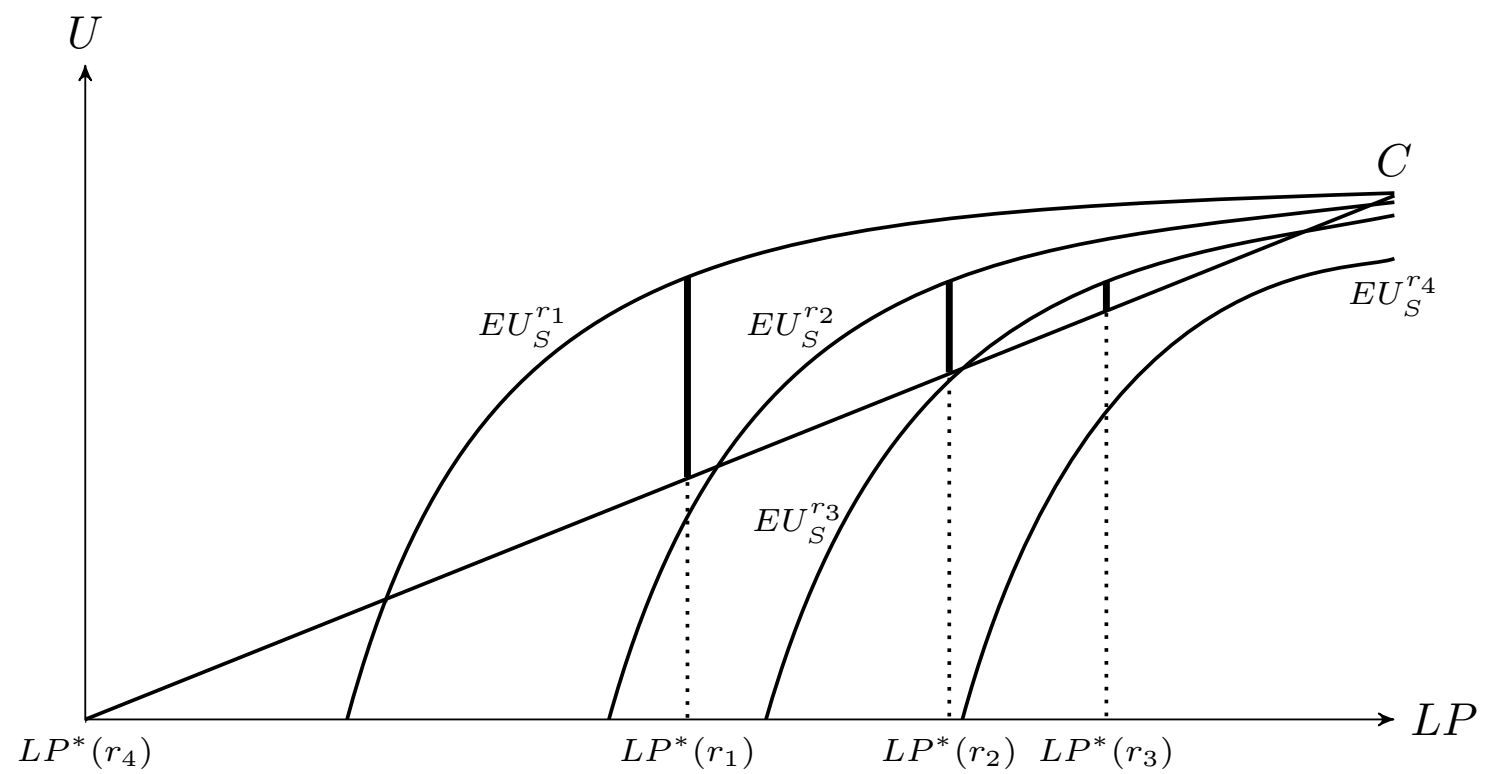

Figure 5: $E U_{S}^{r}$ functions for four requests with different Rates of Imposition $r_{i}$. For each $r \in\left\{r_{1}, r_{2}, r_{3}, r_{4}\right\}$ $L P_{r}^{*}$ represents the optimal amount of politeness for the function $E U_{S}^{r}$.

(12) C: Give me the popcorn!

$\mathrm{M}$ : What is the magic word?

C: Give me the popcorn, PLEASE!

To be more concrete, we assume that Hearer expects a minimum of LP in Speaker's request to even think about allowing Speaker to get the reward. In (12) this minimum amount is the additional politeness marker 'please', without which the mother was not willing to hand out the popcorn. Such a minimum amount LP can be defined as LP amount $p_{0} \in \mathbb{R}^{+}$. Below this value Hearer's probability to grant the request is 0 , which can be formally described as follows: $\forall r \in \mathbb{R}^{+} \exists p_{0} \in \mathbb{R}^{+}: \forall p \in \mathbb{R}^{+}, 0 \leq p \leq p_{0}: \operatorname{Pr}(r \mid p)=0$. The simplest way to add such a $p_{0}$ threshold to the model is to shift the function $E U_{S}^{r}$ by value $p_{0}$ to the right.

The shift of the function to the right changes the optimal amount of linguistic politeness, as shown in Figure 5. Here we consider four requests with different Rates of Imposition $r_{i}$, ordered as follows: $r_{1}<r_{2}<r_{3}<r_{4}$. We also add the implicit assumption that the greater Rate of Imposition $r_{i}$, the greater the shift value $p_{0}$. In other words: if $r_{i}>r_{j}$ then $E U_{S}^{r_{i}}$ is shifted further to the right than $E U_{S}^{r_{j}}$. The figure reveals that the model fulfills the PEP for each $r, r^{\prime} \in\left\{r_{1}, r_{2}, r_{3}, r_{4}\right\}$ under the assumption that $r_{4} \geq r_{\delta}$ (note: $r_{\delta}$ is the threshold value as given in Definition 1), as we will delineate in what follows. ${ }^{26}$

Let's assume that $r_{4} \geq r_{\delta}$, but $r_{1}, r_{2}$, and $r_{3}$ each are less than $r_{\delta}$. Then the set of optimal strategies $\left\{L P^{*}\left(r_{1}\right), L P^{*}\left(r_{2}\right), L P^{*}\left(r_{3}\right), L P^{*}\left(r_{4}\right)\right\}$ as displayed in Figure 5 is in accordance with the PEP. For $r_{4}$ the best strategy is to say nothing at all: $L P^{*}\left(r_{4}\right)=0$. This fulfills the first condition of the PEP (Definition 1), which is the case, since $r_{4} \geq r_{\delta}$. Furthermore, for all $r, r^{\prime} \in\left\{r_{1}, r_{2}, r_{3}\right\}$ it holds that if $r \geq r^{\prime}$ then $L P^{*}(r) \geq L P^{*}\left(r^{\prime}\right)$. This fulfills the second condition of the PEP (Definition 1), which is the case, since $r_{1}, r_{2}, r_{3}<r_{\delta}$.

\footnotetext{
${ }^{26}$ Note that a Rate of Imposition $r$ is assumed to be greater than $r_{\delta}$, if and only if the function $E U_{S}^{r}$ does not surpass the cost function $C$ for any LP amount $p$. As illustrated by Figure 5, this is only the case for function $E U_{S}^{r_{4}}$, therefore only $r_{4} \geq r_{\delta}$. The next section includes a mathematical calculation for the value $r_{\delta}$.
} 
All in all, the presented examples (Figure 4 and Figure 5) point to the fact that the PEP model makes the desired prediction, namely that use of LP in requests is in accordance with the PEP. In the next section we present an explicit mathematical formalization and analysis of the PEP model to prove that it makes the desired prediction.

\section{Mathematical Analysis of the PEP Model}

The first step of the mathematical analysis is to formally define the functions. The expected utility function $E U_{S}^{r}: \mathbb{R}^{+} \rightarrow \mathbb{R}^{+}$is supposed to be defined as a strictly monotonously increasing function with a decreasing slope. Additionally, the function is shifted to the right by a value $p_{0} \in \mathbb{R}^{+}$that is proportional to the Rate of Imposition value $r$. A function that has such properties can simply be defined as $E U_{S}^{r}(p)=\left\{\begin{array}{cl}\left(p-p_{0}\right)^{\frac{1}{2}} & \text { if } p>p_{0} \\ 0 & \text { else }\end{array}\right.$ with $p, p_{0} \in \mathbb{R}^{+}$and $p_{0} \propto r$. For the sake of simplicity, instead of shifting the function by a value $p_{0}$ that is proportional to $r$, we can shift the function by the value $r$ itself. Note that it doesn't change the desired properties of the function. ${ }^{27}$ Thus, the expected utility function can be defined as follows:

Definition 2 (Speaker's Expected Utility Function) Given a situation where Speaker requests a good or service from Hearer quantified as a value of Rate of Imposition $r \in \mathbb{R}^{+}$. Let $p \in \mathbb{R}^{+}$be the amount of LP of Speaker's request. Then Speaker's expected utility function $E U_{S}^{r}: \mathbb{R}^{+} \rightarrow \mathbb{R}^{+}$ can be defined as follows:

$$
E U_{S}^{r}(p)=\left\{\begin{array}{cl}
(p-r)^{\frac{1}{2}} & \text { if } p>r \\
0 & \text { else }
\end{array}\right.
$$

Furthermore, the cost function $C: \mathbb{R}^{+} \rightarrow \mathbb{R}^{+}$is a linear function with a slope value $\beta \in \mathbb{R}^{+}$:

Definition 3 (Speaker's Cost Function) Given a situation where Speaker requests a good or service from Hearer. Let $p \in \mathbb{R}^{+}$be the amount of LP of Speaker's request. Then Speaker's cost function $C: \mathbb{R}^{+} \rightarrow \mathbb{R}^{+}$can be defined as follows:

$$
C(p)=\beta \times p
$$

whereby $\beta \in \mathbb{R}^{+}$

In this way we can define Speaker's total payoff ${ }^{28}$ function $T P: \mathbb{R}^{+} \rightarrow \mathbb{R}$ as the difference between expected utility and cost:

Definition 4 (Speaker's Total Payoff) Given a situation where Speaker requests a good or service from Hearer quantified as a value of Rate of Imposition $r \in \mathbb{R}^{+}$. Let $p \in \mathbb{R}^{+}$be the amount of LP of Speaker's request. Then Speaker's total payoff function $T P^{r}: \mathbb{R}^{+} \rightarrow \mathbb{R}$ can be defined as follows:

$$
T P^{r}(p)=E U_{S}^{r}(p)-C(p)
$$

Now Speaker's optimal strategy $L P^{*}$ would be to use an amount of LP $p$ that maximizes the total payoff, therefore it can be defined as follows:

\footnotetext{
${ }^{27}$ Simply because each variable is proportional to itself.

${ }^{28}$ Note that the total payoff can also be negative, therefore the function's co-domain is not restricted to nonnegative numbers.
} 
Definition 5 (Speaker's Optimal Strategy) Given a situation where Speaker requests a good or service Hearer quantified as a value of Rate of Imposition $r \in \mathbb{R}^{+}$. Let $T P^{r}(p)$ be Speaker's total payoff for amount of $L P p \in \mathbb{R}^{+}$as defined in Definition 4. Then Speaker's optimal amount of politeness $L P^{*}(r)$ can be defined as follows:

$$
L P^{*}(r)=\max _{p}\left(T P^{r}(p)\right)
$$

To find value $L P^{*}(r)$ that maximizes $T P^{r}(p)$, we have to differentiate between the cases where $p \leq r$ and where $p>r$. Let's label the optimal value for the lower part, the case $p \leq r$, with $L P_{l}^{*}(r)$. Note that for this domain range it holds that $T P^{r}(p)=0-C(p)=-\beta \times p$ (since $\left.E U_{S}^{r}(p)=0\right)$. Furthermore, note that this value is always negative except for the case when $p=0$ : then $T P^{r}(p)=0$. Thus, for the domain range $p \leq r$ the optimal amount of politeness $L P_{l}^{*}(r)=0$ and its total payoff is $T P^{r}\left(L P_{l}^{*}(r)\right)=T P^{r}(0)=-C(0)=-(\beta \times 0)=0$.

For the upper part - the case where $p>r$ - we label the optimal value as $L P_{u}^{*}(r)$. Here, the situation is slightly more complicated, since $T P^{r}(p)$ is defined as $E U_{S}^{r}(p)-C(p)=(p-r)^{\frac{1}{2}}-\beta \times p$. To find the maximum $L P_{u}^{*}(r)$ we have to set the first derivation to zero (13) and also check if the second derivation is negative (14):

(13) For the case $p>r$, where $T P^{r}(p)=(p-r)^{\frac{1}{2}}-\beta \times p$, find $L P_{u}^{*}(r)$ that maximizes $T P^{r}(p)$ :

$$
\begin{aligned}
& T P^{r}\left(L P_{u}^{*}(r)\right)^{\prime}=0 \\
\Longleftrightarrow & \left(\left(L P_{u}^{*}(r)-r\right)^{\frac{1}{2}}-\beta \times L P_{u}^{*}(r)\right)^{\prime}=0 \\
\Longleftrightarrow & \frac{1}{2}\left(L P_{u}^{*}(r)-r\right)^{-\frac{1}{2}}-\beta=0 \\
\Longleftrightarrow & \left(L P_{u}^{*}(r)-r\right)^{-\frac{1}{2}}=2 \beta \\
\Longleftrightarrow & L P_{u}^{*}(r)-r=\left(\frac{1}{2 \beta}\right)^{2} \\
\Longleftrightarrow & L P_{u}^{*}(r)=\frac{1}{4 \beta^{2}}+r
\end{aligned}
$$

(14) For the case $p>r$, where $T P(p)=(p-r)^{\frac{1}{2}}-\beta \times p$, show that $T P\left(L P_{u}^{*}(r)\right)^{\prime \prime}<0$ :

$$
\begin{aligned}
& T P\left(L P_{u}^{*}(r)\right)^{\prime \prime}<0 \\
\Longleftrightarrow & \left(\left(L P_{u}^{*}(r)-r\right)^{\frac{1}{2}}-\beta \times L P_{u}^{*}(r)\right)^{\prime \prime}<0 \\
\Longleftrightarrow & -\frac{1}{4}\left(L P_{u}^{*}(r)-r\right)^{-\frac{3}{2}}<0 \\
\Longleftrightarrow & -\frac{1}{4}\left(L P_{u}^{*}(r)-r\right)<0 \\
\Longleftrightarrow & -\left(L P_{u}^{*}(r)-r\right)<0 \\
\Longleftrightarrow & r-L P_{u}^{*}(r)<0 \\
\Longleftrightarrow & r<L P_{u}^{*}(r)
\end{aligned}
$$

In a nutshell, (13) and (14) show that $L P_{u}^{*}(r)=\frac{1}{4 \beta^{2}}+r$ is the amount of LP that maximizes the total payoff of Speaker for the domain range $p>r$. Furthermore, we know for the domain 
range $p \leq r$ that the optimal value $L P_{l}^{*}(r)=0$ results in a total payoff $T P^{r}\left(L P_{l}^{*}(r)\right)=0$. From this we can conclude that in cases where $T P^{r}\left(L P_{u}^{*}(r)\right)<0$ then $L P_{l}^{*}(r)$ is the optimal strategy: to use no LP at all. Consequently, in cases where $T P^{r}\left(L P_{u}^{*}(r)\right)>0$ then $L P_{u}^{*}(r)=\frac{1}{4 \beta^{2}}+r$ is the optimal strategy. Therefore, it is essential to find out under what conditions $T P^{r}\left(L P_{u}^{*}(r)\right)<0$, i.e. under what conditions the best strategy is to use no LP at all. This is done in (15):

(15) Under what conditions is $T P^{r}\left(L P_{u}^{*}(r)\right)<0$ :

$$
\begin{aligned}
& T P\left(L P_{u}^{*}(r)\right)<0 \\
\Longleftrightarrow & \left(L P_{u}^{*}(r)-r\right)^{\frac{1}{2}}-\beta \times L P_{u}^{*}(r)<0 \\
\Longleftrightarrow & \left(\frac{1}{4 \beta^{2}}+r-r\right)^{\frac{1}{2}}-\beta \times\left(\frac{1}{4 \beta^{2}}+r\right)<0 \\
\Longleftrightarrow & \left(\left(\frac{1}{2 \beta}\right)^{2}\right)^{\frac{1}{2}}-\beta \times \frac{1}{4 \beta^{2}}-\beta \times r<0 \\
\Longleftrightarrow & \frac{1}{2 \beta}-\frac{1}{4 \beta}-\beta \times r<0 \\
\Longleftrightarrow & \frac{1}{4 \beta}-\beta \times r<0 \\
\Longleftrightarrow & \frac{1}{4 \beta^{2}}-r<0 \\
\Longleftrightarrow & \frac{1}{4 \beta^{2}}<r
\end{aligned}
$$$$
\text { with } L P_{u}^{*}(r)=\frac{1}{4 \beta^{2}}+r
$$

Note that (15) shows that if reward value $r>\frac{1}{4 \beta^{2}}$ then the optimal strategy is to use amount of politeness $L P_{l}^{*}(r)$, thus to use no LP at all. In other words, $\frac{1}{4 \beta^{2}}$ represents the threshold value $r_{\delta}$ (see Definition 1 , Condition (1)). Below this value the optimal strategy is to use an optimal amount of politeness of $L P_{u}^{*}(r)=\frac{1}{4 \beta^{2}}+r$. Thus, the overall optimal amount of politeness $L P^{*}(r)$ with respect to the given model is given as follows:

Theorem 1 Given a situation where Speaker requests a good or service from Hearer quantified as a value of Rate of Imposition $r \in \mathbb{R}^{+}$. Let $T P^{r}(p)$ be Speaker's total payoff for using amount of LP $p \in \mathbb{R}^{+}$as defined in Definition 4. Then Speaker's optimal amount of politeness $L P^{*}(r)$ is given as follows:

$$
L P^{*}(r)=\max _{p}\left(T P^{r}(p)\right)=\left\{\begin{array}{cl}
\frac{1}{4 \beta^{2}}+r & \text { if } r<\frac{1}{4 \beta^{2}} \\
0 & \text { else }
\end{array}\right.
$$

Note that Theorem 1 shows that the first condition of the PEP (Definition 1) is fulfilled, since for the given model we found the threshold $r_{\delta}=\frac{1}{4 \beta^{2}}$ for which holds: $\forall r \geq r_{\delta}: L P^{*}(r)=0$. Now we have to show that also the second condition also holds, namely that $\forall r, r^{\prime}\left\langle r_{\delta}\right.$ : if $r>r^{\prime}$ then $L P^{*}(r)>L P^{*}\left(r^{\prime}\right)$. In (16) we show that $r>r^{\prime} \Leftrightarrow L P^{*}(r)>L P^{*}\left(r^{\prime}\right)$, given that $\beta$ is a fixed value, thus invariant over $r, r^{\prime}$ : 
(16) Proof that $\forall r, r^{\prime}<r_{\delta}: r>r^{\prime} \Leftrightarrow L P^{*}(r)>L P^{*}\left(r^{\prime}\right)$

$$
\begin{aligned}
& L P^{*}(r)>L P^{*}\left(r^{\prime}\right) \\
& \Longleftrightarrow \frac{1}{4 \beta^{2}}+r>\frac{1}{4 \beta^{2}}+r^{\prime} \\
& \Longleftrightarrow r>r^{\prime} \\
& \text { with } L P^{*}(r)=\frac{1}{4 \beta^{2}}+r
\end{aligned}
$$

\section{Discussion of the Model}

As we were able to show in the last section, the PEP model makes the desired predictions, namely that the use of linguistic politeness for requests is in accordance with the PEP. But note that the PEP model presented here also makes a number of assumptions related to communicative behavior that call for clarification.

One point to clarify is the fact that Speaker's expected utility $E U_{S}^{r}(p)$ might be invariant over $r$, thus its slope does not depend on the magnitude of the request $r$. In Section 9 we delivered a formal rationalization for it. Here we present an example: you go to a funfair with only 3 Euro in your pocket. First you stop at a raffle ticket booth where by buying a 3 Euro ticket you have a $50 \%$ chance of winning an item worth 10 Euro. Then you stop at a second raffle ticket booth where by buying a 3 Euro ticket you have a $25 \%$ chance for an item worth 20 Euro. Your expected utility to try winning one or the other item is exactly the same: 10 Euro $\times 0.5=20$ Euro $\times 0.25=5$ Euro. In other words, the expected utility is the same in both cases and therefore invariant over the final reward, which might be either 10 Euro in the first and 20 Euro in the second lottery. Note further, we only claimed that the function CAN BE invariant over $r$, but not that it necessarily has to be. But this CAN BE is sufficient for arguing that the mechanism of the neediness principle - namely that $r$ lifts the slope of the function - is not appropriate for the PEP model.

The other problem pertains to the very idea of a polite request as defined in the PEP model. One might ask: Why should Hearer grant a request at all, given that Speaker's benefit of utility for getting the request granted is at the same time Hearer's loss of utility for doing it? In gametheoretic terms, such a course of action is essentially a non-cooperative game where Hearer loses whenever he grants a request; thus his best option might appear to never grant a request. In other words, the probability function for Hearer $\operatorname{Pr}_{H}(r \mid p)$ granting a request $r$ would be zero for any amount $p$ of LP Speaker uses. But in our model this probability function is only zero for a range of values $p \leq p_{0}$, whereas there is a positive probability for Hearer to grant a request when $p>p_{0}$. What could make Hearer choose the bad option in a non-cooperative game solely on account of the fact that Speaker pays enough LP?

The PEP model as introduced here represents only Speaker's point of view in making a rational decision by using the right (minimum) amount of LP for getting Hearer to grant a request, but it does not take Hearer's strategic point of view into consideration. It abstracts from the concrete motives Hearer is driven by when making her decision. In other words, the PEP model represents only one side of the coin. We believe that the coin does have two sides, and Hearers also stand to gain something from LP directed to them and from granting the request. We believe so for reasons we explain in section 4: since LP functions like a currency, it implies it has purchasing power for both sides of the interaction. As we have stressed repeatedly, we remain noncommittal on what this value consists in - this is to be determined in future empirical studies. But we have noted that the possible mechanisms: (a) polite language influences social status (decreases status of the sender and increases status of the addressee, Danescu-Niculescu-Mizil et al., 2013), (b) a polite 
request incurs an obligation (direct reciprocity over repeated interactions, e.g. Kreps et al., 1982; Axelrod, 1984), (c) a combination of both, with a role to play by indirect reciprocity/reputation (e.g. Nowak, 2006; Quinley, 2012). Here, however, the PEP model abstracts from all Hearer's motives for making a decision.

A final point to clarify considers Speaker's cost function. One might ask: Does the cost of making a verbal request depend on whether it is granted or not? If we understand the cost of LP as a direct IOU (I owe you) - an emotional debt - in case when a request is granted, then LP would always be costless in cases where it is not granted. It would follow that we need two cost functions depending on the outcome, and one of them is always zero. But if we think of (the main aspect of) costs of LP as Speaker degrading herself a little bit (or paying face), then this self-degrading act involves emotional cost proportionally to LP she uses, but independent of Hearer granting the request or not. ${ }^{29}$ In other words, the cost of LP is invariant over all possible outcomes and only one cost function is needed.

\section{Conclusion and Outlook}

One of the grand problems in understanding language from an evolutionary perspective has been reconciling two major approaches to communication: the cynical approach (Krebs \& Dawkins, 1984) and the cooperative approach, which involves the code model (Bühler, 1934; Jakobson, 1960; Shannon \& Weaver, 1963) as well as Gricean pragmatics (Grice, 1975; Sperber \& Wilson, 1986). The second of these approaches has in-built assumptions of the cooperative character of interaction which - except under very special circumstances, such as kin selection - are not met in the Darwinian world (cf. Searcy \& Nowicki, 2005). In turn, the cynical model makes predictions that are falsified by actual language use, which instead follows Gricean predictions (e.g. the default assumption being that speakers tell the truth rather than lie). As such, the cynical model has been next to useless for describing human language and is absent from the mainstream linguistic theory.

Here, we have pointed to a more general explanatory principle, i.e. alignment of interests, which makes it possible to reconcile these two disparate approaches. A majority of forms of animal communication involve situations of incomplete alignment of interests between the sender and the receiver. In contrast, language has a Gricean nature and operates on the implicit assumption that Speaker and Hearer act as if their interests are aligned (the so-called "platform of trust", Wacewicz, 2015). This is a different way of stating that language is "cooperative", which is in line with the emerging consensus that the evolution of language is intimately related to the evolution of cooperation, and that a successful scenario of language must necessarily take in an evolutionary story about the emergence of cooperation (e.g. Dor et al., 2014). In the last 20 years or so there have been numerous attempts to explain the evolutionary emergence of the very special social niche required for linguistic communication (costly rituals: Knight, 2014; Power, 2014; marriage contracts: Deacon, 1997; kin-selection: Fitch, 2010; cooperative breeding: Hrdy, 2009; shared intentionality: Tomasello, 1999, 2008; or the interdependence hypothesis: Tomasello et al., 2012). From this perspective, it is particularly interesting to find actual examples of human communication which do not call for such extraordinary cooperative assumptions. Here we consider Linguistic Politeness, which we see as a communicative strategy for resolving or at least moderating instances of disaligned interests between interlocutors. This general approach, however, can be extended to deal with other linguistic phenomena, such as indirect speech (Pinker et al., 2008), or preference-dispreference organization of conversational turns (Wootton, 1981; Levinson, 1983; Pomerantz, 1984).

Unlike many facets of language - phonology, syntax, semantics or even pragmatics - linguistic politeness has attracted little attention of evolutionarily minded researchers. We think that this

\footnotetext{
${ }^{29}$ As Quinley (2012, p. 230) puts it: “[...] requests necessarily involve a loss of face to the requester. So X [Speaker] loses face regardless of whether Y [Hearer] helps him or not."
} 
lack of interest - apart from a few isolated attempts (van Rooy, 2003; Żywiczyński, 2012; Wacewicz et al., 2015) - is not dictated by a peripheral status of LP for the description of language, and specifically for language evolution. LP is a universal characteristic of languages but such that is subject to a lot pressure of cultural background, and hence could be a very attractive area for evolutionary modeling. Next, LP is first and foremost a set of interactional strategies, and hence should be easily translated into game-theoretic terms (cf. Quinley, 2011, 2012). We think that the biggest obstacle to an evolutionary approach to LP springs from the problem of defining fitnessrelated consequences of using LP. Note that this problem does not arise with reference to linguistic communication as such - it is easy to see that the transfer of honest information decreases the fitness of the communicator and increases fitness of the receiver. In the case of LP, it is not easy to see what politeness does, in terms of fitness - in particular, the cost of using LP is an open question (see section 4).

In this paper, we suggest a conceptualization in which LP function as a verbal tool for Speaker to contribute expenses (emotional debt, paying face, etc.) for accommodating Hearer's Rate of Imposition. In our view, such a conceptualization is pragmatically realistic; in contrast to van van Rooy's idea of politeness as handicap (2003), it seems to capture the motivational dynamics of conversational interaction. It is then very promising that we have managed to successfully translate this conceptualization into game-theoretic terms and present it in a mathematically sound model.

This is of course just the first step in explicating the evolutionary nature of LP. The model stands in want of empirical verification, and a series of behavioral experiments will be the next step of our research program. Given the operationalization of amount of LP as utterance length, it will be possible to conduct experiments with the use of artificially constructed communication systems, along the lines set in Östman's experiment (1989). Given the strong universalistic underpinnings of our model on the one hand and the culture specific nature of a lot of LP phenomena on the other (Eelen, 2001), it would be particularly interesting to use such an experimental design in a cross-cultural setting. Another major challenge will be extending the model to other speech acts. As was noted, requests seem ideally suited to game-theoretic analysis; it seems that to account for other speech acts - other directives, such as questions, assertives, commissives and expressives - we may have to revise the model by building into it the variable describing the status between Speaker and Hearer, and possibly other variables too (such as e.g. the degree of familiarity between Speaker and Hearer; see Section 5). A revision of this type will of course require the modeling stage and the experimental stage. Finally, on the most general level, we hope that due to the essentially gametheoretic nature of LP our work may help provide a window on linguistic communication at large - a window that will allow us to see the interplay between the Gricean elements of communicative cooperation and the cynical elements sensu Krebs and Dawkins.

\section{References}

Ahern, C. (2015). Cycles and Stability in Linguistic Signaling. Doctoral Dissertation: University of Pennsylvania.

Ahern, C., \& Clark, R. (2017). Conflict, cheap talk, and jespersen's cycle. Semantics and Pragmatics, 10 , article 11.

Asher, N., \& Quinley, J. (2012). Begging questions, their answers and basic cooperativity. In M. Okumura, D. Bekki, \& K. Satoh (Eds.), New Frontiers in Artificial Intelligence - Lecture Notes in Computer Science (Vol. 7258, pp. 3-12). Heidelberg/New York: Springer.

Atkinson, J. M., \& Drew, P. (1979). Order in Court: The Organization of Verbal Interaction in Juridical Settings. London: Macmillan.

Axelrod, R. (1984). The Evolution of Cooperation. New York: Basic Books.

Blum-Kulka, S. (1987). Indirectness and politeness in requests: Same or different? Journal of Pragmatics, $11(2), 131-146$. 
Bousfield, D. (2008). Impoliteness in Interaction. Amsterdam/Philadelphia: John Benjamins.

Brennan, S., \& Ohaeri, J. (1999). Why do electronic conversations seem less polite? the costs and benefits of hedging. In Proceedings, International Joint Conference on Work Activities, Coordination, and Collaboration (WACC '99) (pp. 227-235). San Francisco, CA.

Brown, P., \& Levinson, S. (1978). Universals in language usage: Politeness phenomena. In E. Goody (Ed.), Questions and Politeness: Strategies in Social Interaction (pp. 56-289).

Brown, P., \& Levinson, S. (1987). Politeness: Some Universals in Language Usage. Cambridge: Cambridge University Press.

Bühler, K. (1934). Die Darstellungsfunktion der Sprache. In UTB für Wissenschaft. Band 1159 (pp. 24-33). Stuttgart: G. Fischer.

Burke, M., \& Kraut, R. (2014). Growing closer on facebook: changes in tie strength through social network site use. In Proceedings of the 32nd annual ACM conference on Human factors in computing systems (pp. 4187-4196). New York: ACM Press.

Crawford, V. P., \& Sobel, J. (1982). Strategic information transmission. Econometrica, 50, 1431-1451.

Culpeper, J. (2011). Impoliteness. Using Language to Cause Offence. Cambridge: Cambridge University Press.

Curl, T., \& Drew, P. (2008). Contingency and action: a comparison of two forms of requesting. Research on Language and Social Interaction, 41(2), 129-153.

Danescu-Niculescu-Mizil, C., Sudhof, M., Jurafsky, D., Leskovec, J., \& Potts, C. (2013). A computational approach to politeness with application to social factors. CoRR, abs/1306.6078.

Deacon, T. (1997). The Symbolic Species: The Coevolution of Language and the Brain. New York: W. W. Norton and Company.

Diani, G. (2014). Politeness. In K. Aijmer \& C. Rühlemann (Eds.), Corpus Pragmatics: A Handbook (pp. 169-194). Cambridge: Cambridge University Press.

Dor, D., Knight, C., \& Lewis, J. (2014). Introduction: A social perspective on how language began. In D. Dor, C. Knight, \& J. Lewis (Eds.), The Social Origins of Language (pp. 1-12). Oxford: Oxford University Press.

Duthler, K. (2013). The politeness of requests made via email and voicemail: Support for the hyperpersonal model. Journal of ComputerMediated Communication, 11 (2), 500-521.

Eelen, G. (2001). A Critique of Politeness Theories. Manchester: St. Jerome.

Ehlich, K. (2005). On the historicity of politeness. In S. I. Richard Watts \& K. Ehlich (Eds.), Politeness in Language: Studies in its History, Theory and Practice (pp. 71-107). De Gruyter Mouton.

Ervin-Tripp, S. (1976). Speech acts and social learning. In K. H. Basso \& H. A. Selby (Eds.), Meaning in Anthropology (pp. 123-153). University of New Mexico Press.

Fitch, T. (2010). The Evolution of Language. Cambridge: Cambridge University Press.

Fox, G., Kaplan, J., Damasio, H., \& Damasio, A. (2015). Neural correlates of gratitude. Frontiers in Psychology, 6, 1491. Retrieved from http://journal.frontiersin.org/article/10 .3389/fpsyg.2015.01491 doi: 10.3389/fpsyg.2015.01491

Franke, M. (2009). Signal to Act: Game Theory in Pragmatics. Doctoral Dissertation: Universiteit van Amsterdam.

Goffman, E. (1967). Interaction Ritual: Essays on Face-to-Face Behavior. New York: Doubleday.

Goffman, E. (1983). The interaction order. American Sociological Review, 48, 1-17.

Gordon, D., \& Lakoff, G. (1975). Conversational postulates. In P. Cole \& J. L. Morgan (Eds.), Speech Acts, Syntax and Semantics (pp. 83-106). New York: Academic Press.

Grice, H. (1975). Logic and conversation. In P. Cole \& J. L. Morgan (Eds.), Syntax and Semantics (pp. 41-58). New York: Academic Press.

$\mathrm{Gu}, \mathrm{Y}$. (1990). Politeness phenomena in modern chinese. Journal of Pragmatics, 14(2), 237-257.

Haiman, J. (1983). Iconic and economic motivation. Language, 59(4), 781-819. 
Hrdy, S. (2009). Mothers and Others: The Evolutionary Origins of Mutual Understanding. Cambridge: Belknap Press of Harvard.

Jäger, G. (2008). Evolutionary stability conditions for signaling games with costly signals. Journal of Theoretical Biology, 253(1), 131 - 141.

Jakobson, R. (1960). Linguistik und Poetik. In J. Ihwe (Ed.), Literaturwissenschaft und Linguistik. Ergebnisse und Perspektiven (pp. 142-178). Frankfurt/Main: Athenäum,.

Johnstone, R. A. (1997). The evolution of animal signals. In J. R. Krebs \& N. B. Davies (Eds.), Behavioural Ecology (pp. 155-78). Oxford: Blackwell.

Jucker, A., \& Taavitsainen, I. (2013). English historical pragmatics. Edinburgh: Edinburgh University Press.

Kaplan, D. (1999). What is meaning? Explorations in the theory of Meaning as Use. Brief version - draft 1. University of California, Los Angeles.

Knight, C. (2014). Language and symbolic culture: an outcome of hunter-gatherer reverse dominance. In D. Dor, C. Knight, \& J. Lewis (Eds.), The Social Origins of Language (pp. 228-246). Oxford: Oxford University Press.

Krebs, J., \& Dawkins, R. (1984). Animal signals: mind-reading and manipulation. In J. Krebs \& N. Davies (Eds.), Behavioural Ecology: An Evolutionary Approach (pp. 380-402). Oxford: Blackwell.

Kreps, D., Milgrom, P., Roberts, J., \& Wilson, R. (1982). Rational cooperation in the finitely repeated prisoners' dilemma. Journal of Economic Theory, 27(2), 245-252.

Lakoff, R. (1973). The logic of politeness, or minding your p's and q's. In Papers from the 9th Regional Meeting of the Chicago Linguistic Society (pp. 292-305).

Lakoff, R. (1975). Language and Women's Place. New York: Harper.

Leech, G. (1977). Language and tact. In Series A (p. no. 46). Trier: Universität Trier Linguistic Agency.

Leech, G. (1980a). Explorations in Semantics and Pragmatics. Amsterdam: John Benjamins.

Leech, G. (1980b). Language and tact. In G. Leech (Ed.), Explorations in Semantics and Pragmatics (pp. 79-117).

Leech, G. (1983). The Principles of Pragmatics. London: Longman.

Lee-Wong, S. (1999). Politeness and Face in Chinese Culture. Frankfurt: Peter Lang.

Levinson, S. (1983). Pragmatics. Cambridge: Cambridge University Press.

Lev-On, A., Chavez, A., \& Bicchieri, C. (2010). Group and dyadic communication in trust games. Rationality and Society, 22(1), 37-54.

Lewis, D. (1969). Convention. Cambridge: Harvard University Press.

Mao, L. (1994). Beyond politeness theory: 'face' revisited and renewed. Journal of Pragmatics, $21(5), 451-486$.

Maynard Smith, J. (1982). Evolution and the Theory of Games. Cambridge University Press.

Maynard Smith, J., \& Harper, D. (2003). Animal Signals. Oxford: Oxford University Press.

McEnery, T., Baker, P., \& Cheepen, C. (2001). Lexis, indirectness and politeness in operator calls. Language and Computers, 36(1), 53-69.

Myerson, R. B. (1991). Game Theory: Analysis of Conflict. Harvard University Press.

Nowak, M. A. (2006). Five rules for the evolution of cooperation. Science, 314, 1560-1563.

Östman, J.-O. (1989). Testing iconicity: Sentence structure and politeness. Belgian Journal of Linguistics, 4, 145-163.

Pinker, S., Nowak, M., \& Lee, J. (2008). The logic of indirect speech. Proceedings of the National Academy of Sciences, $105(3)$, 833-838.

Pomerantz, A. (1984). Agreeing and disagreeing with assessments: some features of preferred/dispreferred turn shapes. In J. M. Atkinson \& J. Heritage (Eds.), Structures of Social Action: Studies in Conversation Analysis (pp. 57-101). Cambridge: Cambridge University Press. 
Power, C. (2014). The evolution of ritual as a process of sexual selection. In D. Dor, C. Knight, \& J. Lewis (Eds.), The Social Origins of Language (pp. 196-207). Oxford: Oxford University Press.

Quinley, J. (2011). Politeness and trust games. In D. Lassiter (Ed.), Proceedings of the 2011 ESSLLI Student Session (pp. 143-151). Stanford University.

Quinley, J. (2012). Trust games as a modal request. In D. Lassiter \& M. Slavkovik (Eds.), New directions in Logic, Language and Computation - Lecture Notes in Computer Science (Vol. 7415, pp. 221-233). Heidelberg/New York: Springer.

Quinley, J., \& Ahern, C. (2012). Question of trust. In S. Katrenko \& R. K. Rendsvig (Eds.), Proceedings of the 2012 ESSLLI Student Session (pp. 121-132). Heidelberg/New York: CEUR Workshop Proceedings.

van Rooy, R. (2003). Being polite is a handicap: Towards a game theoretical analysis of polite linguistic behavior. In TARK: Proceedings of the $9^{\text {th }}$ Conference on Theoretical Aspects of Rationality and Knowledge (pp. 45-58). New York: ACM.

Saeed, J. (1997). Semantics. Oxford: Blackwell.

Searcy, W., \& Nowicki, S. (2005). The Evolution of Animal Communication: Reliability and Deception in Signaling Systems. Princeton/Oxford: Princeton University Press.

Searle, J. (1969). Speech Acts. Cambridge: Cambridge University Press.

Searle, J. (1975). Indirect speech acts. In P. Cole \& J. L. Morgan (Eds.), Syntax and Semantics Volume 3: Speech Acts (pp. 59-82). New York/London: Academic Press.

Searle, J., \& Vanderveken, D. (1985). Foundations of Illocutionary Logic. Cambridge: Cambridge University Press.

Shannon, C. E., \& Weaver, W. (1963). The Mathematical Theory of Communication. Urbana/Chicago: University of Illinois Press.

Simmel, G. (1900). Philosophie des Geldes. Leipzig: Duncker \& Humblot.

Sperber, D., \& Wilson, D. (1986). Relevance: Communication and Cognition. Oxford: Blackwell.

Swan, M. (2005). Practical Grammar of English. 3rd edition. Oxford: Oxford University Press.

Terkourafi, M. (2011). From politeness1 to politeness2: Tracking norms of im/politeness across time and space. Journal of Politeness Research, 7(2), 159-185.

Tomasello, M. (1999). The human adaptation for culture. Annual Review of Anthropology, 28, 509-529.

Tomasello, M. (2008). Origins of Human Communication. Cambridge, MA: MIT Press.

Tomasello, M., Melis, A., Tennie, C., Wyman, E., \& Herrmann, E. (2012). Two key steps in the evolution of cooperation: The interdependence hypothesis. Current Anthropology, 53(6), 673-692.

Vilkki, L. (2006). Politeness, face and facework: Current issues. SKY Journal of Linguistics, $19(10), 322-332$.

Wacewicz, S. (2015). The shades of social. a discussion of "the social origins of language". Theoria et Historia Scientiarum, 11, 191-209.

Wacewicz, S., Żywiczyński, P., \& McCrohon, L. (2015). Linguistic politeness from an ethological perspective: Theoretical questions and empirical issues. Theoria et Historia Scientiarum, $11,81-98$.

Watts, R. (2003). Politenes. Cambridge: Cambridge University Press.

Werkhofer, K. (2005). Traditional and modern views: The social constitution and power of politeness. In R. Watts, S. Ide, \& K. Ehlich (Eds.), Politeness in Language: Studies in its History, Theory and Practice (pp. 155-199). Berlin: De Gruyter Mouton.

Wootton, A. (1981). The management of grantings and rejections by parents in request sequences. Semiotica, 37, 59-89.

Zahavi, A. (1975). Mate selection — a selection for a handicap. Journal of Theoretical Biology, $53,205-214$. 
Zahavi, A., \& Zahavi, A. (1997). The Handicap Principle: A Missing Piece of Darwin's Puzzle. New York: Oxford University Press.

Żywiczyński, P. (2010). The Axiology of Spoken Interaction: An Essay on the Organisation of Conversational Behaviour. Toru/'n: Wydawnictwo Naukowe Uniwersytetu Mikołaja Kopernika.

Żywiczyński, P. (2012). An attempt at a proxemic description of politeness from the ethologicalevolutionary perspective. Theoria et Historia Scientiarum, 9, 71-98. 